ARTICLE

Received 3 Apr 2013 | Accepted 19 Sep 2013 | Published 18 Oct $2013 \quad$ DOl: 10.1038/ncomms3641

\title{
Separation of a functional deubiquitylating module from the SAGA complex by the proteasome regulatory particle
}

\author{
Sungsu Lim¹, Jaechan Kwak ${ }^{1}$ Minhoo $\mathrm{Kim}^{1} \&$ Daeyoup Lee ${ }^{1}$
}

Gene expression is an intricate process tightly linked from gene activation to the nuclear export of mRNA. Recent studies have indicated that the proteasome is essential for gene expression regulation. The proteasome regulatory particle binds to the SAGA complex and affects transcription in an ATP-dependent manner. Here we report that a specific interaction between the proteasomal ATPase, Rpt2p and Sgf73p of the SAGA complex leads to the dissociation of the H2Bub1-deubiquitylating module (herein designated the Sgf73-DUBm) from SAGA both in vitro and in vivo. We show that the localization of the Sgf73-DUBm on chromatin is perturbed in rpt2-1, a strain of Saccharomyces cerevisiae that is specifically defective in the Rpt2p-Sgf73p interaction. The rpt2-1 mutant also exhibits impaired localization of the TREX-2 and MEX67-MTR2 complexes and is defective in mRNA export. Our findings collectively demonstrate that the proteasome-mediated remodelling of the SAGA complex is a prerequisite for proper mRNA export.

\footnotetext{
${ }^{1}$ Department of Biological Sciences, Korea Advanced Institute of Science and Technology, Daejeon 305-701, Korea. Correspondence and requests for materials should be addressed to D.L. (email: daeyoup@kaist.ac.kr).
} 
T he $26 \mathrm{~S}$ proteasome is a highly conserved protein-degrading machine composed of a barrel-shaped 20S core particle (CP) that is capped at each end with a 195 regulatory particle (RP) ${ }^{1}$. Whereas the $20 \mathrm{~S} \mathrm{CP}$ has protease activity, the base complex of the 19S RP exhibits chaperone-like activity in addition to proteolytic functions $s^{2,3}$. It has been reported that PA700, the mammalian homologue of the 19S RP, binds to misfolded protein substrates, represses their aggregation and remodels their conformation prior to proteolysis ${ }^{4-6}$. At the end of the $\mathrm{M}$ phase, the cyclin B and cdc2 subunits of cyclin B-Cdc2 kinase are separated by the $26 \mathrm{~S}$ proteasome via a mechanism that does not require proteolysis of the cyclin B substate ${ }^{7}$. In addition, the base complex (that is, the hexameric ATPase component) of the 19S RP has been shown to be involved in gene expression irrespective of its proteolytic activity ${ }^{8,9}$. The non-proteolytic functions of the proteasome include its abilities to modulate ubiquitin-dependent covalent histone modifications ${ }^{10}$ and remodel/stabilize the stalled polymerase complex to support efficient transcriptional elongation ${ }^{11}$. The functions of the $19 \mathrm{~S} R P$ in transcriptional elongation are supported by the observed elongation defects in the yeast 19S RP ATPase mutant strains, SUG1 and SUG2 (refs 10,12). Recently, the 19S RP was suggested to regulate the assembly of the active transcription complex at the promoter ${ }^{13}$ by facilitating the interactions between the Spt-Ada-Gcn5-acetyltransferase (SAGA, a yeast transcriptional coactivator) complex and promoter-bound transcription activators ${ }^{14}$. The same study demonstrated a physical interaction between the 19S RP and the SAGA complex and suggested that the 19S RP may change the biochemical properties of the SAGA complex.

SAGA is a multisubunit complex of $1.8 \mathrm{MDa}$ that coordinates multiple post-translational modifications of histones ${ }^{15}$ to regulate diverse cellular functions, including the acetylation of histone H3 (ref. 16), the recruitment of the RNA polymerase II (RNA PolII) pre-initiation-complex and the deubiquitylation of histone $\mathrm{H} 2 \mathrm{~B}$ mono-ubiquitylation at lysine 123 (H2Bub1) ${ }^{17-19}$. Among the submodules of the SAGA complex, the deubiquitylating module (DUBm), which consists of the Sgf73, Ubp8, Sus1 and Sgf11 subunits ${ }^{20,21}$, is responsible for the deubiquitylation of H2Bub1 (refs 17-19). The DUBm facilitates elongation via the deubiquitylation of $\mathrm{H} 2 \mathrm{Bub} 1$ (ref. 22) and contributes to other aspects of gene expression, such as gene gating and mRNA export $^{23}$. Interestingly, previous studies have suggested that the proteasome may be involved in mRNA export ${ }^{6,24,25}$. For example, Sem1, a component of the 19S RP lid subcomplex, was identified as a functional subunit of the TREX-2 complex ${ }^{26,27}$. The physical interaction between the proteasome and the SAGA complex suggests that the proteasome may induce a conformational change in the SAGA complex through its ATP-dependent chaperone-like activity.

Here we report a novel non-proteolytic function of the 19S RP: it specifically interacts with the SAGA complex to induce the dissociation of an Sgf73p-Ubp8-containing complex that displays deubiquitylation activity. Remodelling of the SAGA complex by the proteasome affects the association of the TREX-2 complex with the nuclear pore complex (NPC), which is essential for mRNA export. Our data further suggest that this proteasomemediated alteration of the SAGA complex is involved in transcription-coupled mRNA export.

\section{Results}

The proteasomal ATPase Rpt2p specifically binds to Sgf73p. Previous studies have demonstrated that there is a physical interaction between the 19S RP and the SAGA complex ${ }^{28}$. To investigate the nature of the interactions between the 19S RP and the SAGA complex subunits, we performed in vitro GST pulldown assays with purified SAGA and GST-fused components of the 19S RP. The SAGA complex bound specifically to Rpt2p (Supplementary Fig. S1a, lane 2 versus lanes 1 and 3-6) but not Pre4p (a subunit of the 20S CP) or Rpn1-12p (non-ATPase 19S RP subunits) (Supplementary Fig. S1a, lanes 7 and 8-18). A previous study on the interaction between the human Rpt $2 p$ homologue, S4, and the human Sgf73p homologue, Sca7 (ref. 29), suggested that Sgf73p may be a binding partner of Rpt2p. To test the potential binding relationship between Rpt $2 p$ and Sgf73p, we used GST pull-down assays with SAGA complexes from the wildtype $(w t)$ and $s g f 73 \Delta$ strains. Our results indicated that Rpt2p specifically interacted with the wt SAGA complex in vitro (Supplementary Fig. S1b, lane 3) but not with the SAGA complex lacking Sgf73p (Supplementary Fig. S1b, lane 6 versus lane 9). As Sgf73p is a subunit of the DUBm, we tested whether other subunits of the DUBm could alter the interaction between Sgf73p and Rpt2p. GST pull-down assays showed that the Sgf73pRpt2p interaction patterns of $u b p 8 \Delta$ and sgf 114 were similar to those observed in $w t$ (Supplementary Fig. S1b, lanes 9 and 12). Thus, the interaction between the $26 \mathrm{~S}$ proteasome and the SAGA complex appears to occur specifically through Sgf73p and Rpt2p.

We next sought to identify the Rpt2p-binding domain for the SAGA complex by performing GST pull-down assays with a series of truncated versions of Rpt2p. Our results revealed that the $\mathrm{N}$-terminal domain, specifically the basic patch of Rpt2p (RKKRK), was responsible for binding the SAGA complex (Supplementary Fig. S1c, lane 9). Based on this finding, we used a trans-replacement method to engineer an Rpt2p mutant lacking the RKKRK patch (designated rpt2-1). Collectively, these data show that the proteasomal ATPase, Rpt2p, specifically binds to Sgf73p of the SAGA complex.

The 19S RP dissociates an Sgf73p-Ubp8p-containing complex. Having shown that the interaction between the 19S RP and the SAGA complex is specific, we next investigated the possible effects of this interaction. We hypothesized that the 19S RP alters the conformation of the SAGA complex via the proteasome's innate ATP-dependent chaperone-like activity ${ }^{3}$. To assess this, we combined purified Spt7-TAP-tagged SAGA complexes from rpt2-1 with proteasomes (26S or 19S) in the presence or absence of ATP. The SAGA complexes were then immobilized in calmodulin resin, and the bound and supernatant fractions were separated. Western blotting was performed with antibodies against CBP (Spt7p), FLAG (Sgf73p), MYC (Ubp8p) and HA (Gcn5p) (Fig. 1). Sgf73p and Ubp8p were detected in the supernatant in the presence of ATP (Figs. 1a, b, lane 4), whereas neither Gcn5p nor Spt7p was detected in the supernatant, regardless of the presence of ATP (Fig. 1a,b, lanes 4 and 5). These data indicate that an Sgf73p-Ubp8p-containing complex is separated from the SAGA complex by the 19S RP in an ATP-dependent manner. Interestingly, both Sgf73p and Ubp8p are subunits of the DUBm.

Identical in vitro experiments using the 19S RP from rpt2-1 (Fig. 1c) showed that Sgf73p and Ubp8p were not detected in the supernatant (Fig. 1c, lane 5). Thus, the protein-protein interaction between Sgf73p and Rpt2p appears to be important for the separation of the Sgf73p-Ubp8p-containing complex from the SAGA complex (Fig. 1c, compare lanes 4 and 5).

Whereas the barrel-shaped 20S CP consists of four stacked rings $^{30}$, the 19S RP can be divided into two subcomplexes ${ }^{31}$ : the base and the lid ${ }^{32}$. The base complex contains four non-ATPase subunits (Rpn1, $-2,-10$ and -13 ) and six AAA-type ATPases (Rpt1-6) that exhibit chaperone-like activity ${ }^{3}$. To identify the subcomplex(es) of the 19S RP that is/are responsible for the separation of the Sgf73p-Ubp8p-containing complex from the 
a
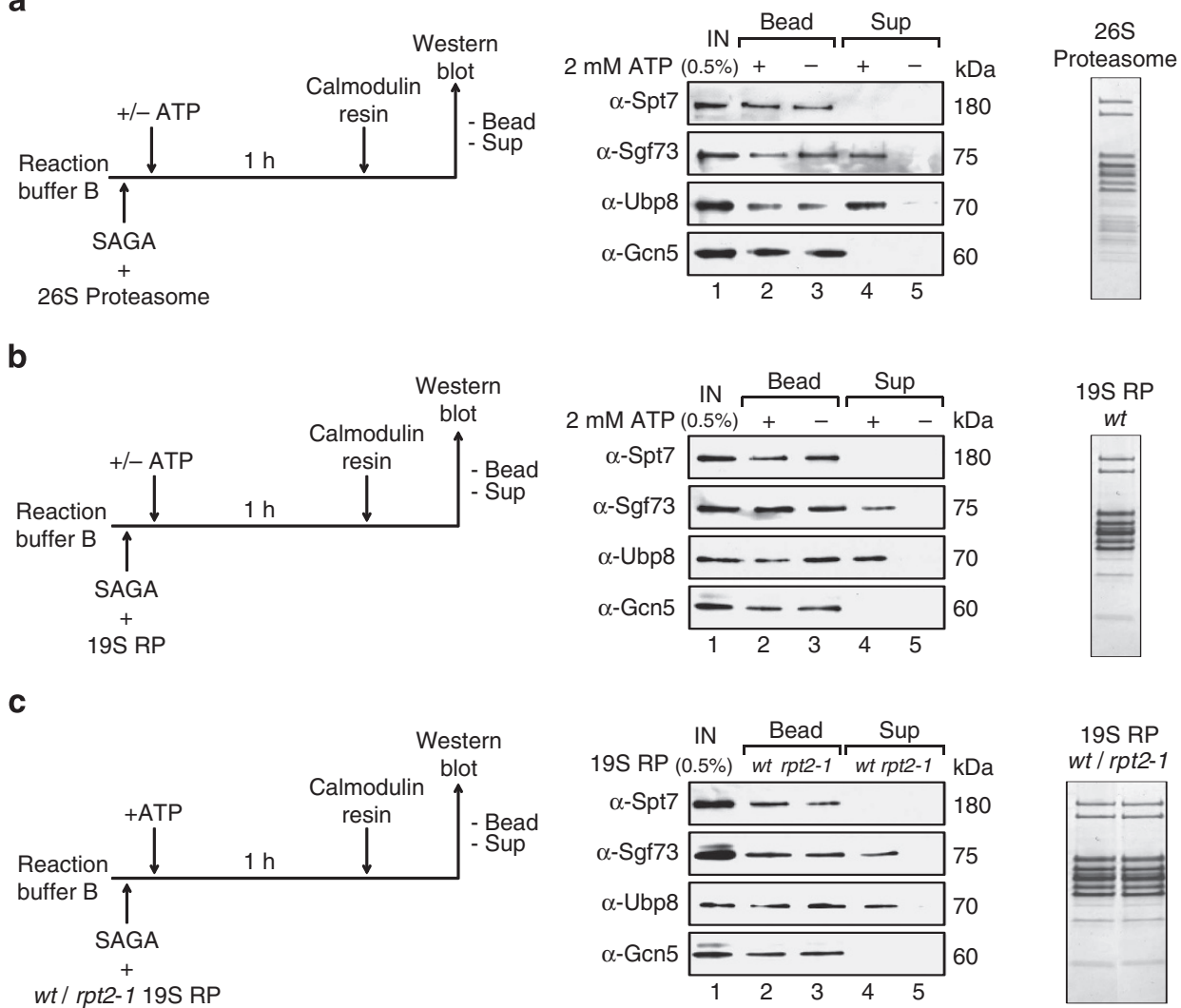

d
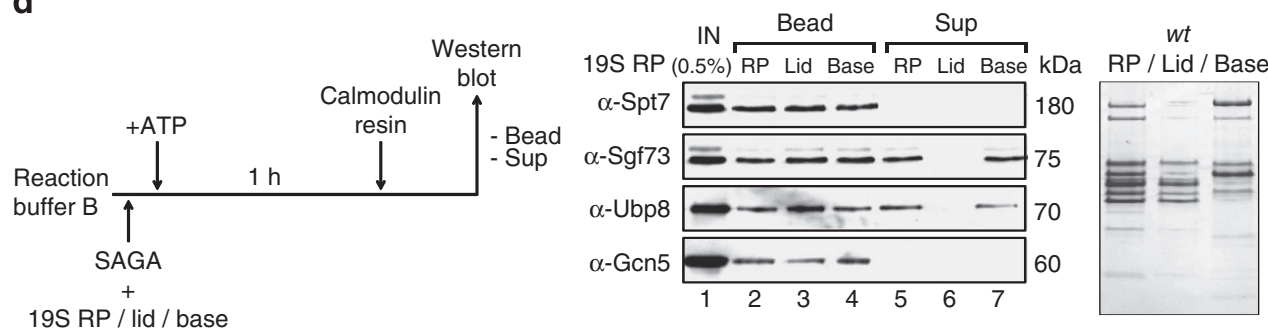

Figure 1 | The 19S RP separates a Sgf73p-Ubp8p-containing complex from the SAGA complex in an ATP-dependent manner. (a,b) Schematic diagram of in vitro experiments (left panel). Spt7-TAP-tagged SAGA complexes purified from rpt2-1 were combined with the 26S proteasome (a) and the 19S RP (b) purified from wt in the presence or absence of $2 \mathrm{mM}$ ATP. After reaction with calmodulin resin, the bound proteins and supernatants were eluted in SDS-PAGE sample buffer and analysed by western blotting with antibodies against CBP (Spt7p), FLAG (Sgf73p), MYC (Ubp8p) and HA (Gcn5p). (c,d) Schematic diagram of in vitro experiments (left panel). Experiments were performed as in (a), except that the Spt7-TAP-tagged SAGA complexes from rpt2-1 were combined with the 19S RP purified from wt and rpt2-1 (c) and the 19S RP, lid complex or the base complex from wt (d). The bound proteins and supernatants were analysed using western blotting with antibodies against CBP (Spt7p), FLAG (Sgf73p), MYC (Ubp8p) and HA (Gcn5p). The purified 26S proteasome, 19S RP, lid complex and the base complex were visualized using Coomassie blue staining (right panel).

SAGA complex, we conducted in vitro experiments with the whole 19S RP, the lid and the base (Fig. 1, right panel). Our results revealed that the base complex alone, but not the lid complex alone, was sufficient to separate the Sgf73p-Ubp8pcontaining complex from the SAGA complex (Fig. 1d, lane 7). These data indicate that the 19S RP dissociates the Sgf73pUbp8p-containing complex from the SAGA complex in an ATPdependent manner through a specific interaction with SAGA.

The proteasome separates a functional Sgf73-DUBm from SAGA. We speculated that the separation of the Sgf73p-Ubp8pcontaining complex via a specific interaction of the proteasome with SAGA could affect the relative abundance of the Sgf73DUBm in the SAGA complexes differently in $w t$ versus rpt2-1 cells. First, we checked the integrity of the SAGA complexes in the $w t$ and $r p t 2-1$ strains, using silver staining of Spt7-TAP-tagged SAGA complexes purified from $w t$ and $r p t 2-1$ (Fig. 2a). Our results indicated that the SAGA complex is intact in rpt2-1.

In terms of abundance, we observed more Sgf73p and Ubp8p in western blots of Spt7-TAP-purified SAGA complexes from rpt2-1 compared with the wt (Fig. 2b, lanes 3 and 4). Quantification of the results from three independent experiments (Fig. 2b, right panel) suggested that the proteasome affects the composition of the SAGA complex in rpt2-1. We questioned whether this difference in abundance was because of altered expression levels of SAGA components in rpt2-1; however, we did not observe any change in the global expression levels of the SAGA complex subunits ${ }^{20,33,34}$ (Sgf73p, Spt20p, Spt8p, Ada1p, Ubp8p, Gcn5p, Ada2p and Sgf11p) in rpt2-1 (Fig. 2c). 
a

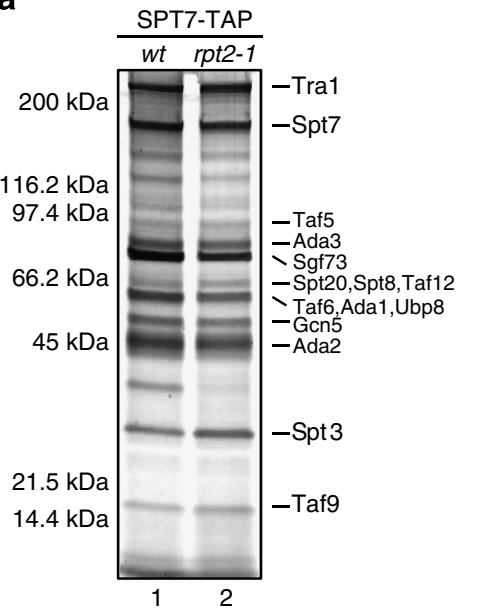

b
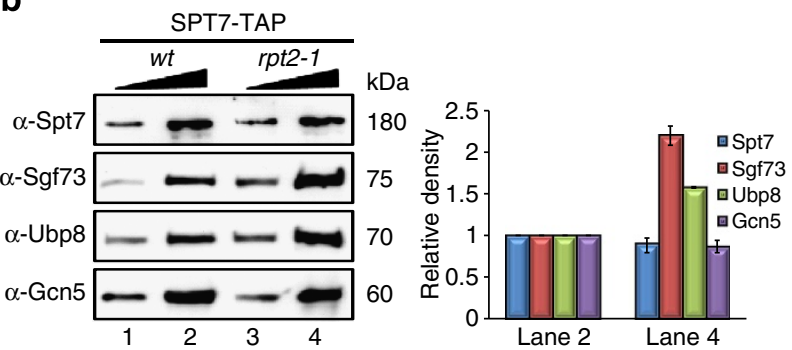

C
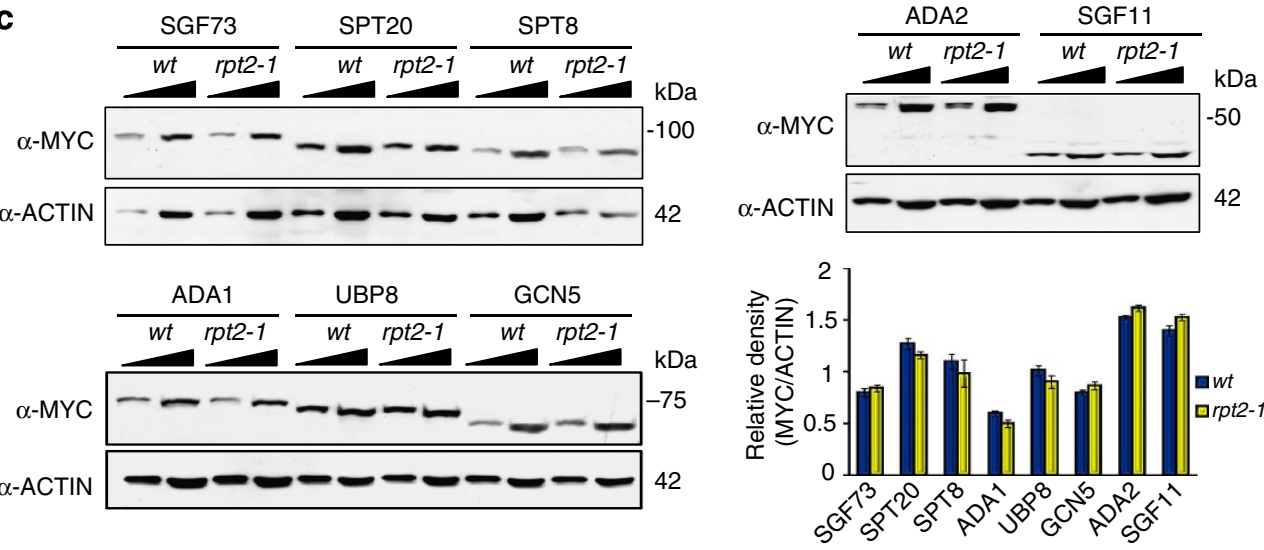

d

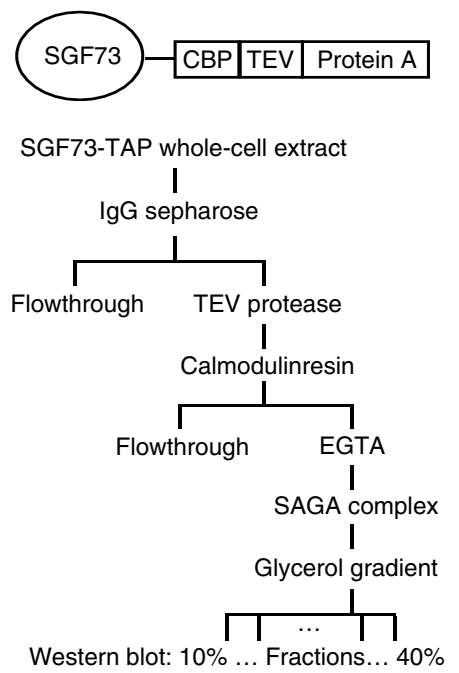

e

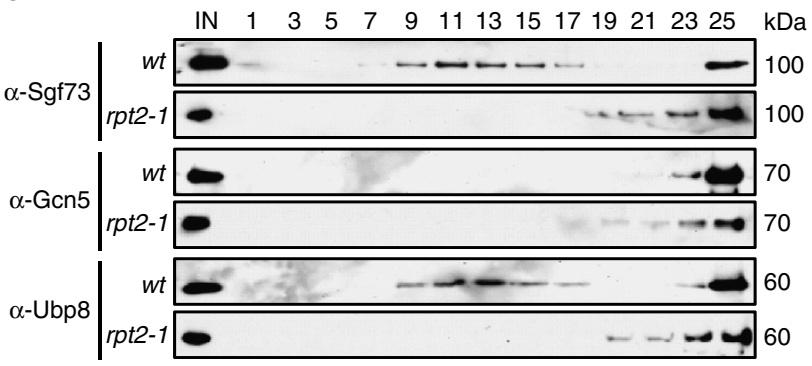

f

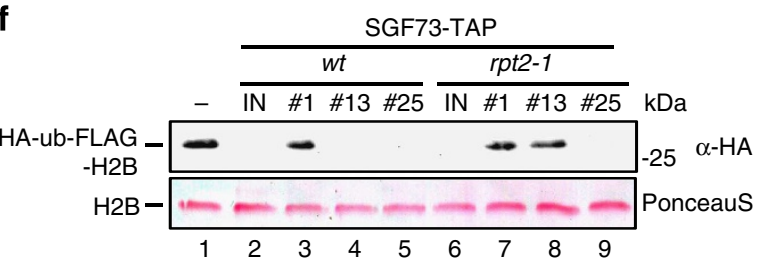

Figure 2 | The Sgf73p-DUBm separated from the SAGA complex has functional H2Bub1 deubiquitylation activity in vivo. (a) Spt7-TAP-tagged SAGA complexes from $w t$ and rpt2-1 were purified using the TAP purification method ${ }^{67}$ and visualized using silver staining. Protein molecular weight standards are indicated. (b) Twofold serially diluted samples of Spt7-TAP-tagged SAGA complexes purified from wt and rpt2-1 were analysed using western blotting with antibodies against CBP (Spt7p), FLAG (Sgf73p), MYC (Ubp8p) and HA (Gcn5p). The blots shown are representative of three independent experiments. Western blot signals were quantified using the Image J software $(\mathrm{NIH})$, and standard deviations are shown by error bars. (c) MYC-tagged wt and rpt2-1 cells were grown in YPD at $30^{\circ} \mathrm{C}$, and total cell extracts were analysed using SDS-PAGE and western blotting using a monoclonal anti-MYC antibody and an anti-ACTIN antibody (control). The quantified results and standard deviations are graphed in the right panel. Western blot signals were quantified using the Image J software $(\mathrm{NIH})$, and standard deviations from four independent experiments are shown by error bars. (d) Schematic diagram of in vitro experiments. (e) Sgf73-TAP-tagged SAGA complexes purified from wt and rpt2-1 were fractionated by 10-40\% glycerol gradient centrifugation, and the fractions were analysed using western blotting with antibodies against CBP (Sgf73p), MYC (Ubp8p) and HA (Gcn5p). IN, input. (f) In vitro deubiquitylation assays of three glycerol gradient fractions (fractions 1, 13 and 25) were conducted using HA-ub-FLAG-H2B as the substrate. Western blotting was performed with an antibody against HA. Mock-treated H2Bub1 substrate was used as the input. 
Quantification of the results from three independent experiments supported our conclusion (Fig. 2c, right panel). Our data suggest that the changes in the SAGA components arise via a posttranslational mechanism.

As the Sgf73p-Rpt2p interaction was found to trigger the separation of the Sgf73p-Ubp8p-containing complex from the SAGA complex in vitro (Fig. 1), we examined whether this separation occurs through a specific interaction of Sgf73p and Rpt $2 p$ in vivo. We performed linear $10-40 \%$ glycerol gradient separations of Sgf73-TAP-tagged SAGA complexes purified from $w t$ and rpt2-1 (Fig. 2d). After centrifugation, fractions were collected and analysed with the help of western blotting using antibodies against CBP (Sgf73p), HA (Ubp8p) and MYC (Gcn5p) (Fig. 2e). The Sgf73p-Ubp8p-containing complex, but not Gcn5p, was detected in fractions 9-15 from the $w t$ samples (Fig. 2e, top panel). In the $r p t 2-1$ samples, however, Sgf73p and Ubp8p were both absent from fractions 9-15; instead, they were found at the bottom of the gradient (in fraction 25), most likely along with the intact SAGA complexes. These data demonstrate that the proteasome separates the Sgf73p-Ubp8p-containing complex from the SAGA complex both in vitro and in vivo.

The DUBm ${ }^{20,21}$ of the SAGA complex is responsible for deubiquitylating histone H2Bub1 (refs 17-19). Owing to the similarities in the composition of the DUBm and the separated Sgf73p-Ubp8p-containing complex, we hypothesized that the dissociated Sgf73p-Ubp8p-containing complex could have deubiquitylation activity. To examine the deubiquitylating ability of the Sgf73p-Ubp8p-containing complex ${ }^{35-37}$, we performed deubiquitylation assays with fractions 1,13 and 25 from the glycerol gradient assay, using HA-ub-FLAG-H2B as the substrate, and examined the results using western blotting with an antibody against HA. Fraction 13 of the $w t$ samples displayed normal H2Bub1-deubiquitylating activity, whereas that from rpt2-1 did not (Fig. 2f, compare lanes 4 and 8). Thus, the Sgf73pUbp8p-containing complex separated from the SAGA complex has functional deubiquitylation activity. In the SAGA complex, Sgf73p stimulates the deubiquitylation of H2Bub1, and both Sgf73p and Ubp8p are necessary for full DUBm protease activity $^{35}$. This deubiquitylation activity is not only because of the simple dissociation of Ubp8p from the SAGA complex but also involves the release of the Sgf73-DUBm. This finding raises the possibility that the separated complex may be the DUBm of the SAGA complex. As a putative DUBm of the SAGA complex, we designated the dissociated Sgf73p-Ubp8p-containing complex the 'Sgf73p-DUBm.'

As SAGA is a Gcn5-dependent histone acetyltransferase (HAT) complex ${ }^{16}$, we also employed core histones ${ }^{38}$ to examine HAT activity in fractions 1, 13 and 25 (Supplementary Fig. S2a) and in purified Spt7-TAP-tagged SAGA complexes from $w t$ and $r p t 2-1$ (Supplementary Fig. S2b). Our results revealed that the SAGA complexes of $r p t 2-1$ and $w t$ displayed similar HAT activity levels, indicating that the SAGA complex in the rpt2-1 mutant shows an in vitro histone $\mathrm{H} 3$ acetyltransferase activity similar to that of $w t$.

The proteasome shows intact ubiquitin-dependent proteolysis. As the altered composition of the SAGA complexes may be because of changes in the proteolytic activity of the proteasome, we assessed the integrity and activity of the $26 \mathrm{~S}$ proteasome from $w t$ and $r p t 2-1$. We first examined the molecular integrity of the 19S RP, using purified TAP-tagged-Rpn1p from the $w t$ and $r p t 2-1$ strains. Silver staining showed that the affinity-purified 19S RP complexes from $w t$ and rpt2-1 had nearly identical protein composition patterns (Fig. 3a), indicating that the integrity of the 19S RP is maintained in rpt2-1.
To analyse the enzymatic activities of the proteasome, we performed LLVY assays with the fluorogenic substrate, SucLLVY-AMC, and purified Rpn1-TAP-tagged 26S proteasomes from $w t, r p t 2-1$ and pre1-1/2-2 (a proteasomal mutant in the beta subunit of the 20S CP and defective in the chymotrypsin-like activity of the $26 \mathrm{~S}$ proteasome $\mathrm{e}^{39,40}$ ). We resolved the affinitypurified $26 \mathrm{~S}$ proteasome from $w t$ and $r p t 2-1$ on native gels and visualized the active proteasomal complexes using an in-gel activity assay ${ }^{41}$ and found that the signal patterns of the ATPdependent peptidase activities of the proteasomes from $w t$ and rpt2-1 were essentially the same (Fig. 3b). We additionally measured the peptidase activity of the proteasome by liquid-based LLVY assays ${ }^{42}$ over a time course of $0,10,20$ and $30 \mathrm{~min}$. Our data showed that the proteasomes purified from $w t$ and $r p t 2-1$ had similar peptidase activities (Fig. 3c). These results indicate that the global enzymatic activity and assembly pattern of the proteasome are maintained in the rpt2-1 strain.

To examine the ubiquitin-dependent proteolytic function of the $26 \mathrm{~S}$ proteasome, we assessed polyubiquitinated protein degradation activity in the $w t$ and rpt2-1 strains in vivo (Fig. 3d) and in vitro (Fig. 3e). We used the proteasome temperature-sensitive $(t s)$ mutant, cim3-1, which is defective in one of the 19S ATPase subunits (Rpt6p) and shows severely reduced proteasome activity at $37^{\circ} \mathrm{C}$ (ref. 43). $w t, r p t 2-1$ and cim3-1 cells were collected at different times, and total cell extracts were subjected to western blotting with the FK2 antibody against polyubiquitin. Our results revealed that high-molecularmass polyubiquitinated proteins accumulated to much lower degrees in $w t$ and $r p t 2-1$ cells compared with cim 3-1 cells grown at the restrictive temperature (Fig. 3d, lanes 7-10 versus lanes 11 and 12). Next, using highly polyubiquitinated-Sic1 ${ }^{\mathrm{PY}}$ as a substrate $^{44,45}$, we performed in vitro measurements of the proteolytic activities of $26 \mathrm{~S}$ proteasomes purified from $w t$ and $r p t 2-1$. We found that polyubiquitinated-Sic1 ${ }^{\mathrm{PY}}$ was efficiently degraded within $5 \mathrm{~min}$ by $26 \mathrm{~S}$ proteasomes purified from both $w t$ and rpt2-1 (Fig. 3e, lanes 3 and 6). Thus, the proteasomemediated degradation of polyubiquitinated proteins does not appear to be defective in the rpt2-1 mutant.

The Sgf73-DUBm accumulates near promoter regions in rpt2-1. Our results indicated that the proteasome dissociates the Sgf73-DUBm from SAGA. Therefore, blocking the release of Sgf73-DUBm in rpt2-1 cells is expected to cause retention of Sgf73-DUBm at the sites of SAGA complex recruitment, such as promoters or transcription factor-binding sites. We analysed the relative levels of the Sgf73-DUBm on chromatin in $w t$ and rpt2-1. We used chromatin immunoprecipitation (ChIP) assays to examine the chromatin association of various SAGA components with the constitutively expressed PMA1 gene in $w t$ and $r p t 2-1$. Our results revealed that the promoter region of PMA1 was more highly occupied by Sgf73p, Ubp8p and Sgf11p in $r p t 2-1$ versus $w t$ (Fig. 4a, up to twofold enrichment), whereas the levels of Spt20p, Spt8p, Adalp, Gcn5p and Ada2p at the promoter and open reading frame $(\mathrm{ORF})$ regions of $P M A 1$ were unaltered or slightly reduced in rpt2-1 compared with the $w t$ (Supplementary Fig. S3). Similar results were obtained from ChIP assays performed with the ADH1 and PYK1 genes (Fig. 4b,c, and Supplementary Fig. S3). These data further confirm that the proteasome affects the composition of SAGA and suggests that intact proteasomes separate the Sgf73-DUBm from the SAGA complex to near promoter regions in $w t$ cells, whereas the undissociated Sgf73DUBm remains bound to the SAGA complex at promoter regions in rpt2-1 cells.

To examine the effects of rpt2-1 on the recruitment of RNA polymerase II (RNA PolII) to chromatin, we used ChIP to 
a

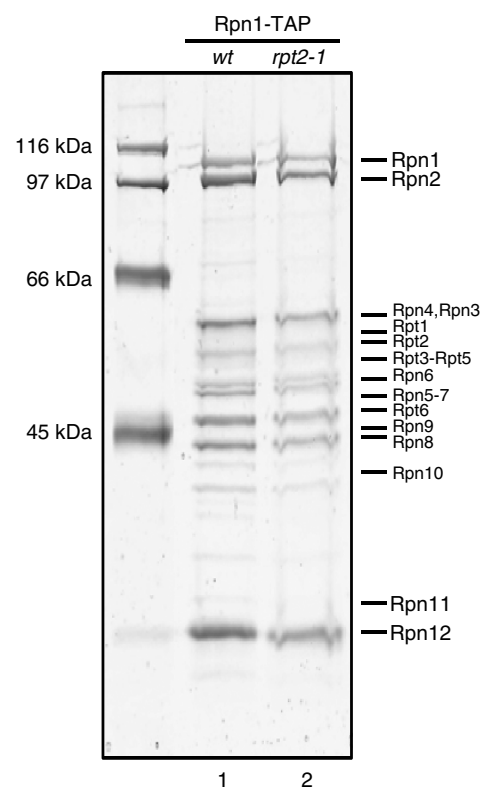

b

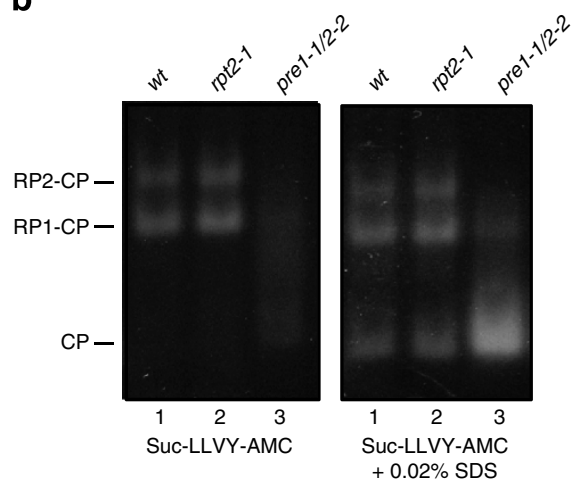

C

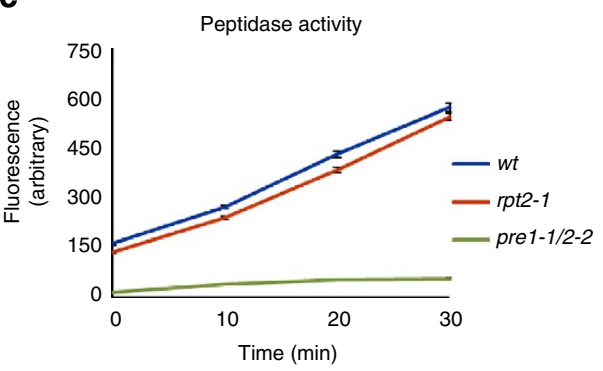

e

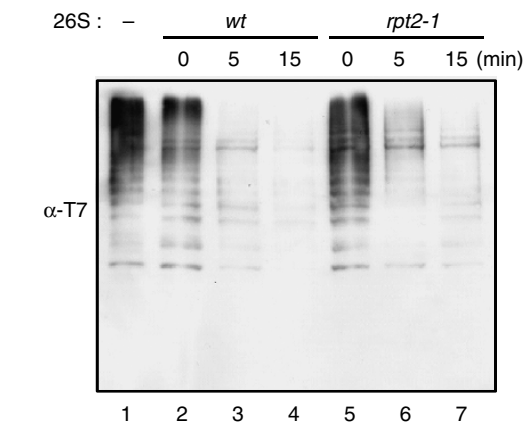

d

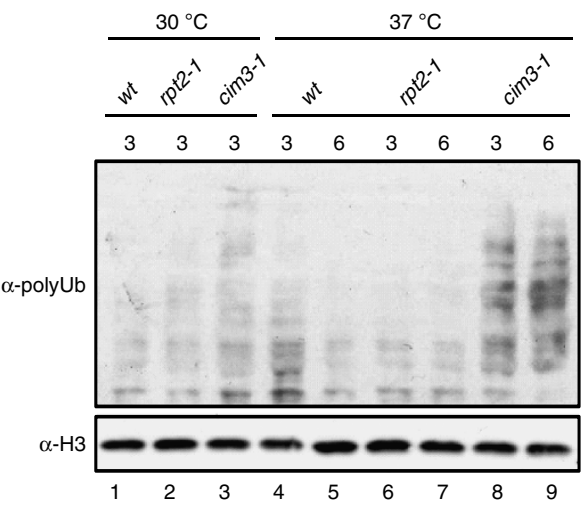

Figure 3 | The composition of the 19S RP and the proteolytic activity of the 26S proteasome are unaltered in rpt2-1. (a) Rpn1-TAP-tagged 19S RPs from wt and rpt2-1 were purified and visualized using silver staining. Protein molecular weight standards are indicated. (b-c) The 26S proteasomes in rpt2-1 show normal peptidase activity and assembly. (b) Purified proteasomes from wt and rpt2-1 were resolved on native gels and stained for hydrolytic activity using the fluorogenic substrate, Suc-LLVY-AMC, in the absence and presence of $0.02 \%$ SDS. RP2-CP, RP1-CP and CP refer to doubly capped proteasomes, singly capped proteasomes and the core particle, respectively. (c) Purified proteasomes from wt and rpt2-1 were incubated with the fluorogenic substrate, Suc-LLVY-AMC and peptidase activity was measured with a fluorescence spectrophotometer. Error bars represented standard deviations of three independent experiments. (d) Polyubiquitinated proteins that are not accumulated in rpt2-1. wt, rpt2-1 and cim3-1 cells were grown to the log phase in YPD at $30^{\circ} \mathrm{C}$ and then shifted to $37^{\circ} \mathrm{C}$. The cells were collected at the indicated times, and total cell extracts were subjected to western blotting with FK2 antibodies against polyubiquitin. H3 was used as a control. (e) The $26 \mathrm{~S}$ proteasome in rpt2-1 has intact degradation activity towards polyubiquitinated proteins in vitro. The $26 \mathrm{~S}$ proteasomes from wt or rpt2-1 were incubated with polyubiquitinated $\operatorname{Sic} \mathrm{P}^{\mathrm{PY}}$ at $25^{\circ} \mathrm{C}$. The reaction was terminated by the addition of SDS-loading buffer at the indicated times, and the reaction mixture was subjected to western blotting with an antibody against T7.

measure the cross-linking of 8WG16 (which detects hypophosphorylated forms of RNA PolII (ref. 46)) to actively transcribed regions of PMA1. We observed similar levels of recruitment in $w t$ and rpt2-1 (Supplementary Fig. S4a). We also used ChIP analysis to examine the recruitments of the proteasome to the PMA1 gene promoter (Supplementary Fig. S4b) and found that the $w t$ and rpt2-1 strains exhibited similar occupancy levels of proteasomal ATPases (for example, Rpt2p and Rpt6p) on the PMA1 promoter regions. Thus, the difference in the recruitment of the SAGA complex to chromatin does not appear to be associated with the recruitment of RNA PolII or the proteasome.
Remodelling of SAGA is critical for proper TREX-2 trafficking. An association between the TREX-2 (also called THSC) complex and the NPC is critical for the proper export of mRNAs from the nucleus to the cytoplasm, where they are translated into proteins by the cytoplasmic translational machinery ${ }^{23,47}$. Previous studies have shown that the interaction between TREX-2 (refs 48-51) and the NPC is mediated by Sgf7 $3 \mathrm{p}^{23}$. TREX- 2 mediates the association of active genes to the nuclear pores by binding to both the nuclear face of the NPC and the chromatin-bound SAGA comple ${ }^{52}$. As Sgf73p was enriched at the promoter regions in $r p t 2-1$, we tested whether the separation of the Sgf73-DUBm is 
a
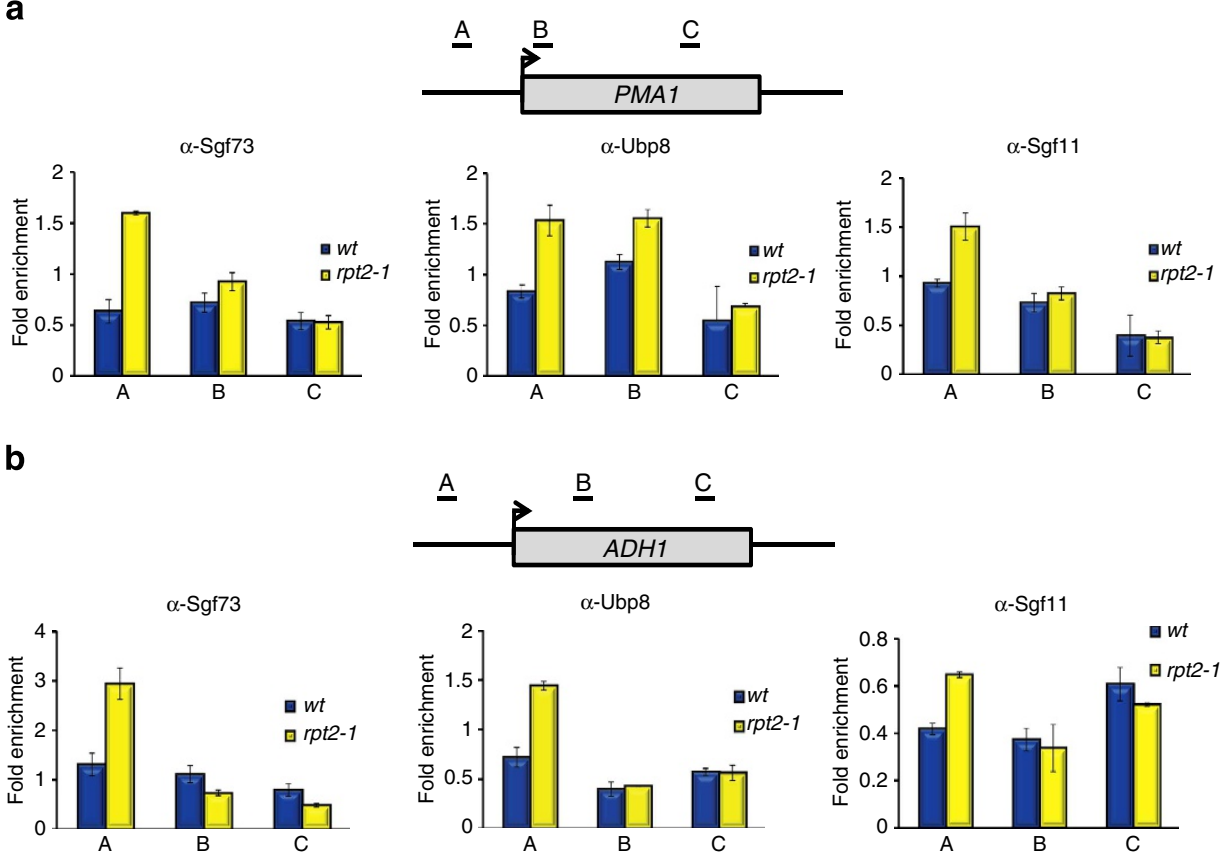

C
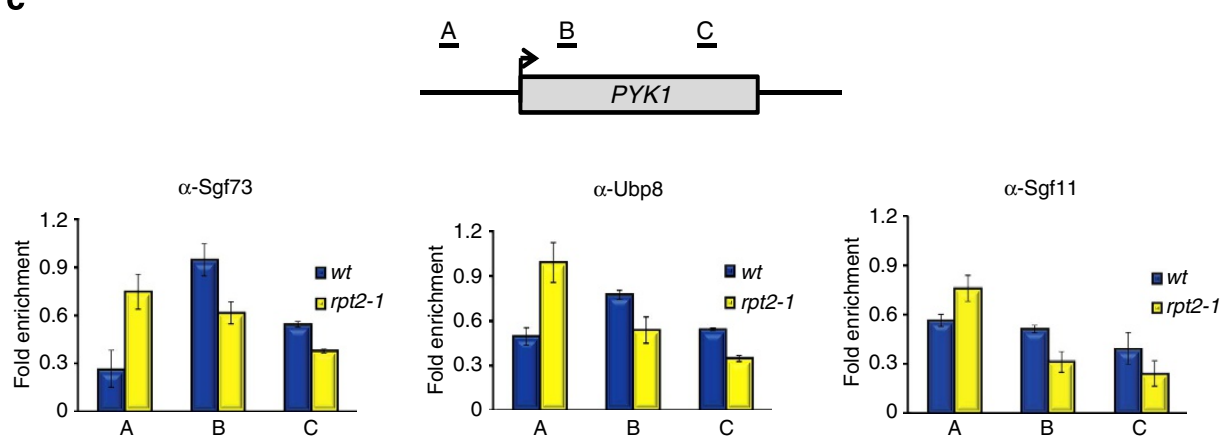

Figure 4 | The Sgf73p-DUBm shows increased enrichment near the gene promoters in rpt2-1. The relative abundances of Sgf73p, Ubp8p, Gcn5p and Sgf11p on the promoter regions of the PMA1 (a), ADH1 (b) and PYK1 (c) genes in wt and rpt2-1 were measured using ChIP assays followed by q-PCR using the indicated primers. ChIP assays and q-PCR were performed on five independent extracts. Standard deviations are shown by error bars.

important for the trafficking of TREX-2 between chromatin and the NPC. Prior to this analysis, we first examined the integrity of the TREX-2 complex and the SAGA-TREX-2 association in rpt2-1 and observed that these parameters were similar between $w t$ and rpt2-1 (Supplementary Fig. S5). Next, we performed ChIP assays with antibodies against Sac3p and Thp1p to examine the targeting of chromatin-bound TREX-2 to the NPC in the PMA1 genes of the $w t, r p t 2-1$ and $s a c 3 \Delta$ strains. Surprisingly, the promoter region of PMA1 was highly occupied by Sac $3 p$ and Thplp in rpt2-1 (Fig. 5a, up to fourfold and twofold, respectively) compared with the $w t$. Similar results were obtained from ChIP assays using the $A D H 1$ and PYK1 genes (Supplementary Fig. S6), suggesting that the enrichment of TREX-2 at promoters was not specific to the PMA1 gene. These data demonstrate that the proteasome-mediated remodelling of the SAGA complex is important for the proper association between the TREX-2 complex and the NPC.

As the proteasome-mediated separation of the Sgf73-DUBm from the SAGA complex was found to be important for the TREX-2-NPC interaction, we investigated the subcellular localization of TREX-2 in rpt2-1 using green-fluorescent protein (GFP)-fused Sac3p (the largest component of TREX-2) (Fig. 5b). Sac3-GFP was localized predominantly to the nuclear periphery in $w t$ cells (Fig. 5b, wt) ${ }^{23}$, but in $r p t 2-1$ and $s g f 73 \Delta$, it was partly mislocalized to the nuclear periphery and the cytoplasm (Fig. 5b, rpt2-1 and sgf734). Furthermore, in the sus14 strain, mislocalization of TREX-2 to the cytoplasm was further enhanced (Fig. 5b, sus 14$)^{23}$. These results indicate that proteasome-mediated remodelling of the SAGA complex is required for the correct targeting of Sac3p to the NPC. Additionally, we analysed the subcellular localization of another TREX-2 component (Thp1p) and a component of the NPC (Nuplp) in rpt2-1 using GFP-fused Thplp and -Nup1p, respectively. Similar to Sac3-GFP ${ }^{23}$, Thp1GFP was localized predominantly to the nuclear periphery of $w t$ cells; in rpt2-1, however, it was partly mislocalized to the nuclear periphery and the cytoplasm. Thus, mislocalization of the TREX-2 complex is not only specific to Sac3p but also correlates with the mislocalization of Thp1p. In contrast, Nup1GFP was localized to the nuclear side of the NPC in both $w t$ and rpt2-1 cells ${ }^{53}$. These results suggest that the correct targeting of Nuplp to the NPC is unaffected in rpt2-1. Meanwhile, the sus1A strain showed enhanced mislocalization of Thp1-GFP to the cytoplasm but exhibited normal localization of Nup1-GFP. Therefore, the proteasome-mediated remodelling of the SAGA complex does not seem to affect the translocation of nucleoporin to the NPC (that is, the last step of mRNA export). 
a

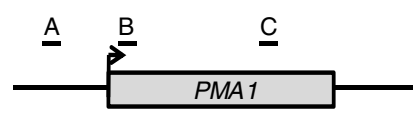

$\alpha-\operatorname{Sac} 3$

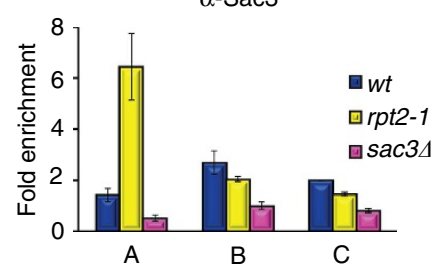

$\alpha-M Y C(T h p 1)$

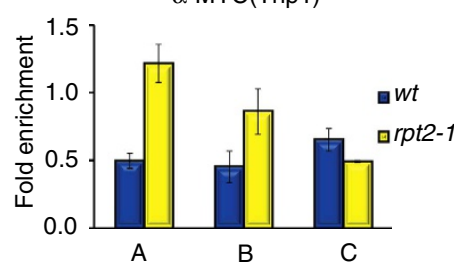

b

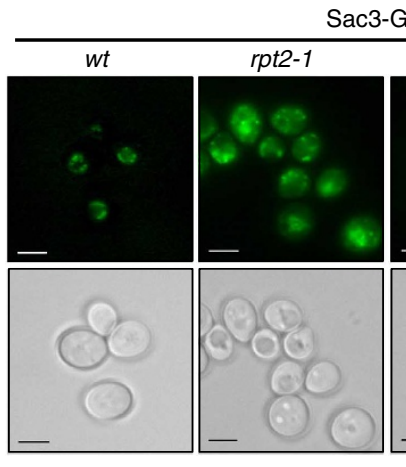

Thp1-GFP
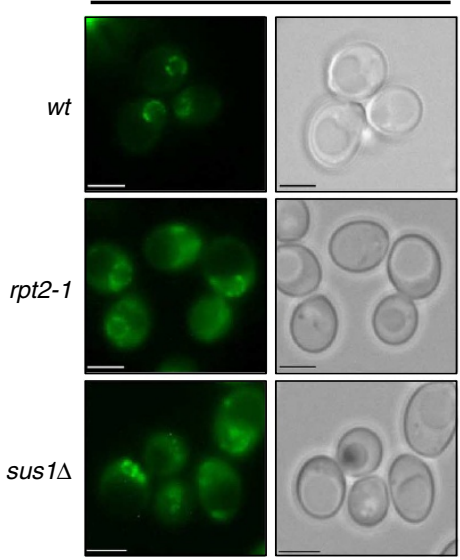

d
C
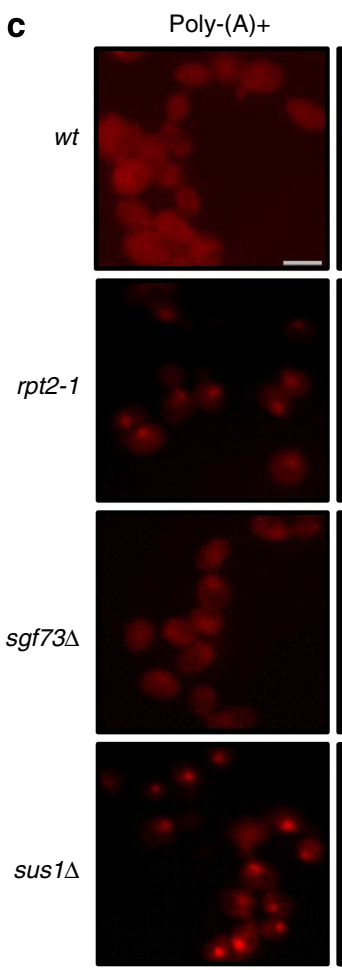

DNA
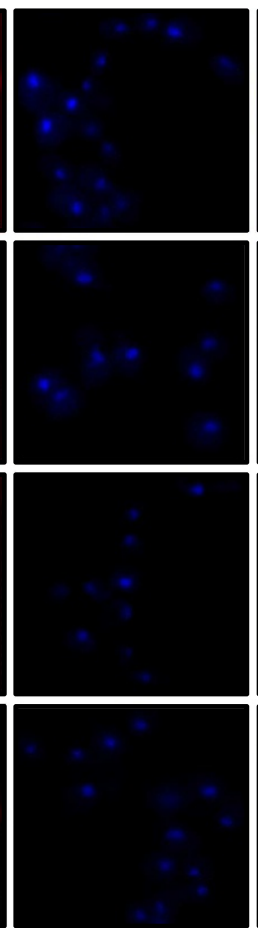

Merge
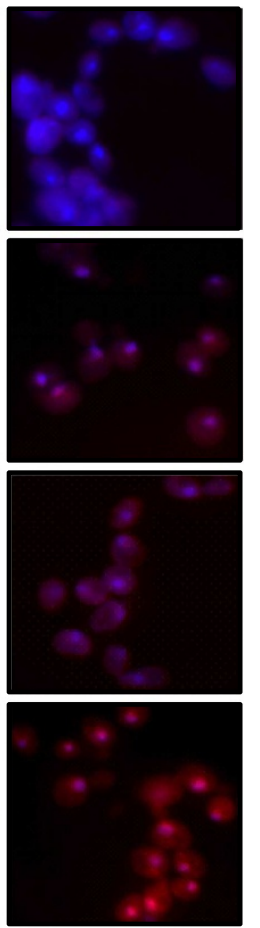

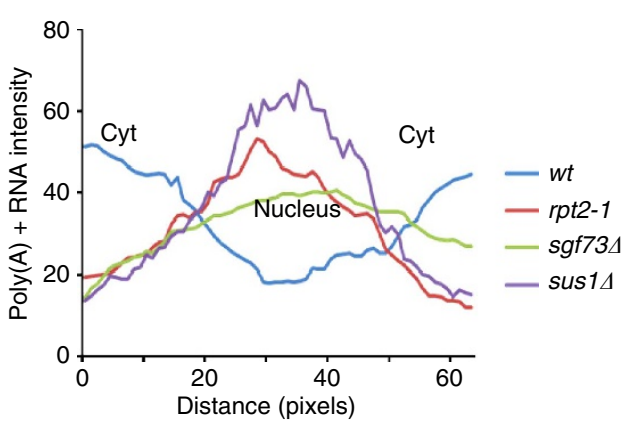

Figure 5 | Remodelling of the SAGA complex by the Sgf73p-Rpt2p interaction is critical for efficient mRNA export and the TREX-2-NPC association in vivo. (a) TREX-2 is enriched on the promoter region of the PMA1 gene in rpt2-1. ChIP assays of the PMA1 gene in wt, rpt2-1 and sac34 were performed using antibodies against Sac3p and MYC (Thp1p). ChIP assays and q-PCR were performed on five independent extracts. Standard deviations are shown by error bars. (b) The subcellular localization of TREX-2 is affected in rpt2-1. The subcellular localizations of Sac3-GFP, Thp1-GFP and Nup1-GFP in wt, rpt2-1, sgf734 and sus14 are shown, as captured using fluorescence microscopy and Nomarski photographs. Scale bar, $5 \mu \mathrm{m}$. (c) Export of mRNA is defective in rpt2-1. Analyses of nuclear mRNA export in wt, rpt2-1, sgf73 4 and sus14 are shown. Poly (A) + RNA was detected using FISH with Cy3-labelled oligo(dT) probes and DNA was stained with 4',6-diamidino-2-phenylindole (DAPI). Scale bar, $10 \mu \mathrm{m}$. (d) Quantitative graph of poly(A) + RNA intensity (assessed using FISH analysis) in wt, rpt2-1, sgf734 and sus14 cells is shown. Pairs of cells with nuclei of the same scan width (as determined with DAPI staining) were used for measurements. The cytoplasm (Cyt) and nucleus are indicated. 
To confirm that rpt2-1 cells are defective in mRNA export, fluorescence in situ hybridization (FISH) analysis was performed using Cy3-labelled oligo (dT) probes (Fig. 5c, left panel). This analysis revealed a significant accumulation of poly-(A) + RNA in the nucleus of $r p t 2-1$ cells compared with the $w t$ cells. Deletion of SUS1 was previously shown to trigger significant deficits in mRNA export ${ }^{50}$, whereas deletion of $S G F 73$ induced less accumulation of poly-(A)+ RNA in the nucleus (Fig. 5c). Quantification of the nuclear poly-(A) + RNA intensity levels in $w t, r p t 2-1$, sgf73 $\Delta$ and sus1 $\Delta$ cells (Fig. 5d) demonstrated that the proteasome-mediated separation of the Sgf73-DUBm from the SAGA complex is important for efficient mRNA export.

rpt2-1 genetically interacts with alleles of Mex67-Mtr2. In the mRNA export pathway, mRNAs form complexes with proteins to generate export-competent ribonucleoparticles ${ }^{54}$ that are translocated through the NPC with the help of the Mex67-Mtr2 complex. The Mex67-Mtr2 complex is an essential mRNA export factor that promotes the translocation of ribonucleoparticles by associating with FG repeat nucleoporins ${ }^{55}$. Sac3p interacts directly with Mex67-Mtr2 via these FG repeats ${ }^{48}$ and functions in the translocation of Mex67p-Mtr2p through the NPC ${ }^{51}$. In the sac3 4 mutant, the Mex67-Mtr2 complex is mislocalized; this results in synthetic lethality when combined with ts mutants of the Mex67-Mtr2 complex ${ }^{51,56}$.

Here we investigated the subcellular localization of the Mex67Mtr2 complex in rpt2-1 using GFP-fused Mex67p and Mtr2p. Mex67p and Mtr2p were found in the nuclear envelope in $w t^{57}$ (Fig. 6a, wt). In rpt2-1, however, they were partially mislocalized to the cytoplasm and less significantly to numerous cytoplasmic foci

a

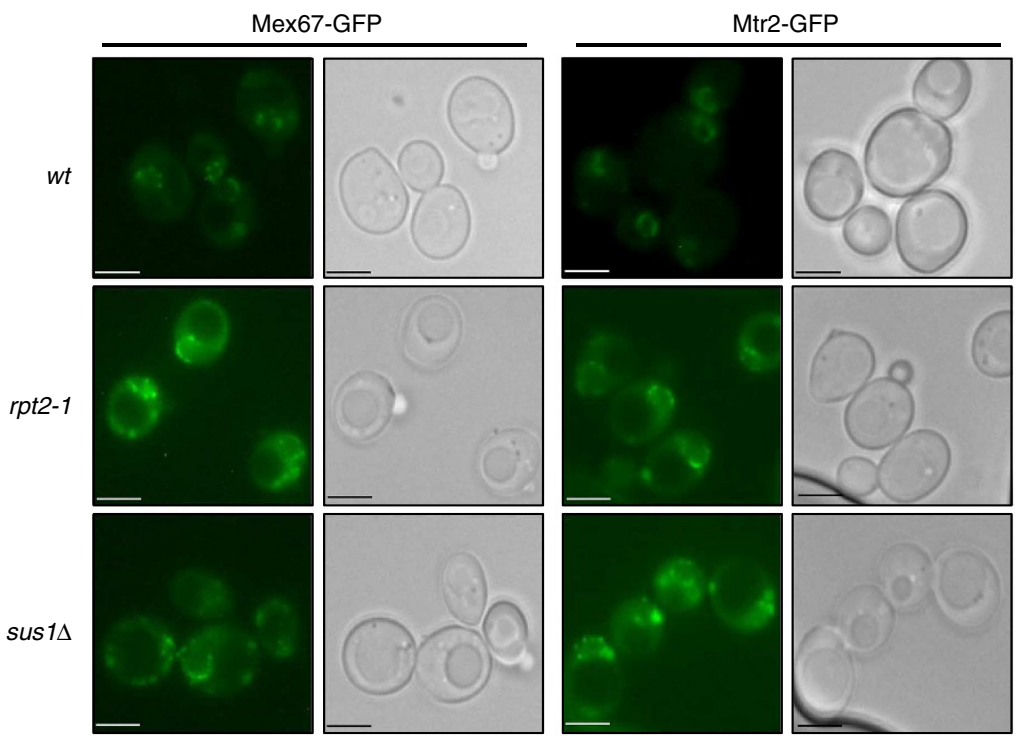

b $\operatorname{mex} 67-5$
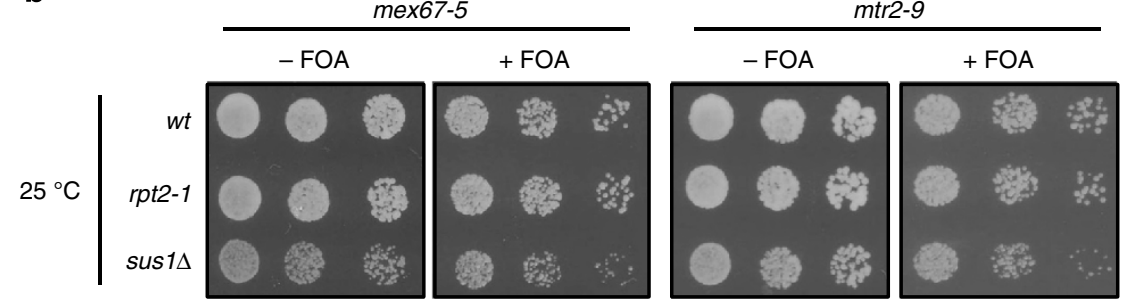

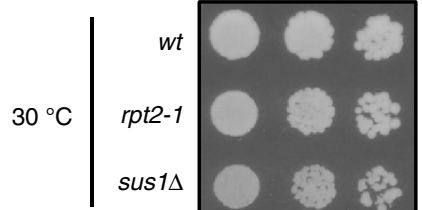
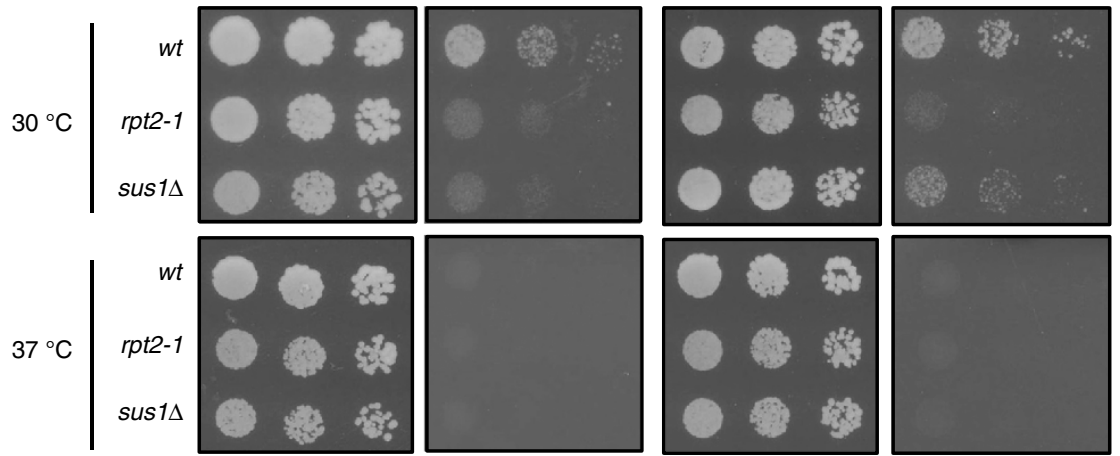

Figure 6 | The Mex67-Mtr2 complex is mislocalized and interacts genetically with Mex67-Mtr2 mutant alleles in rpt2-1. (a) The subcellular localization of the Mex67-Mtr2 complex is affected in rpt2-1. The subcellular localizations of Mex67-GFP and Mtr2-GFP in wt, rpt2-1 and sus14 are shown, as visualized using fluorescence microscopy and Nomarski photographs. Scale bar, $5 \mu \mathrm{m}$. (b) The rpt2-1 mutant interacts genetically with mex67-5 and mtr2-9. The ts mutant alleles mex67-5 and mtr2-9 were transformed into the wt, rpt2-1 and sus14 shuffle strains of wt MEX67 and MTR2 plasmid, respectively. Precultures were diluted in the SC medium and equivalent amounts of cells ( $1 / 6$ diluted) were spotted on synthetic complete (SC) plates with and without 5-FOA (0.075\%). wt MEX67 and MTR2 plasmids were shuffled out from cells when plated on SC plates with 5-FOA. Plates were incubated for 3 days at 25,30 and $37^{\circ} \mathrm{C}$. 
(Fig. 6a, rpt2-1). Therefore, our data indicate that the proteasomemediated remodelling of the SAGA complex has a direct effect on the proper localization of the Mex67-Mtr2 complex.

To understand the physiological importance of the rpt2-1 allele in terms of the mRNA export pathway, we asked whether rpt2-1 interacts genetically with other factors involved in mRNA export, such as MEX67 or MTR2. We used the previously reported 5 -fluoro-orotic acid (FOA) screening method to assess synthetic lethality between alleles ${ }^{56}$. We transformed the $t s$ mutant alleles mex67-5 (ref. 56) or $m t r 2-9$ (ref. 57) into the wt, rpt2-1 and sus14 shuffle strains of $w t$ MEX67 or MTR2 plasmid, respectively. We performed spotting assays on plates with or without 5-FOA, and the plates were incubated at 25,30 and $37^{\circ} \mathrm{C}$. Cells grown on 5 FOA $(-)$ plates showed no apparent growth defects at both permissive and restrictive temperatures. However, cells undergoing plasmid shuffling on 5-FOA $(+)$ plates showed strong $t s$ phenotypes when grown at restrictive temperature: $t s$ phenotypes of mex67-5 and $m$ tr2-9 alleles appeared from all cells grown on 5 -FOA $(+)$ at $37^{\circ} \mathrm{C}$ (refs 56,57$)$. In contrast, all mutant cells grown on 5 -FOA $(+)$ at $25^{\circ} \mathrm{C}$ showed growth pattern similar to $w t$. Interestingly, double mutants of rpt2-1 with either mex67-5 or mtr2-9 showed significant growth defects compared with $w t$ when grown on $5-\mathrm{FOA}(+)$ at $30^{\circ} \mathrm{C}$ (Fig. 6b). Furthermore, as demonstrated in a previous study, double mutant of sus $1 \Delta$ and mex67-5 showed significant growth defects at $30^{\circ} \mathrm{C}$ compared with $w t$ on $5-\mathrm{FOA}(+)$ plates ${ }^{50}$. Such enhanced growth retardation on $5-\mathrm{FOA}(+)$ plates indicates synthetic lethality between mex67-5/mtr2-9 and $r p t 2-1$ alleles. This suggests a strong genetic interaction between rpt2-1 and mRNA export factors MEX67 and MTR2.

\section{Discussion}

Our findings collectively suggest that the proteasome alters the composition of the SAGA complex through the Rpt2p-Sgf73p interaction and that the 19S RP ATP dependently dissociates the Sgf73-DUBm from the SAGA complex in vitro. Previous studies

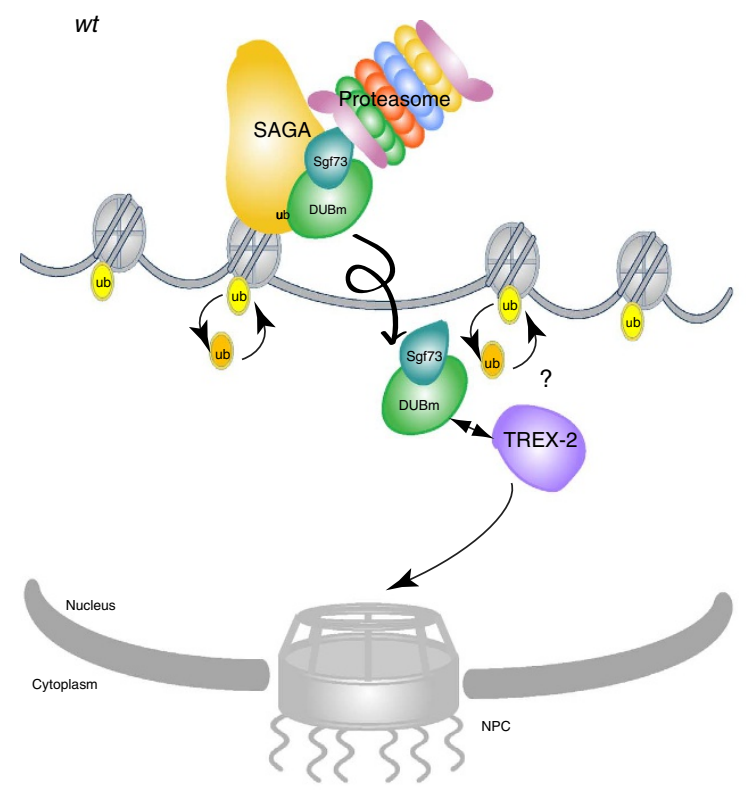

detected Ubp8p at the promoter and ORF regions of GAL1 (ref. 22) and showed that Ubp8p interacts specifically with RNA PolII and functions as a component of the SAGA complex at ORFs during transcriptional elongation ${ }^{20,22}$. The high occupancy levels of Sgf73p, Ubp8p and Sgf11p at the tested promoter regions in rpt2-1 cells suggest that Ubp8p or the Sgf73-DUBm may have specific roles in transcriptional elongation. It is highly likely that the Sgf73-DUBm of the SAGA complex interacts with ORFs along with phosphorylated RNA PolII and that this is required for transcriptional elongation and is important for the recycling of $\mathrm{H} 2 \mathrm{Bub} 1$. Changes in the amount of H2Bub1 in the ORF regions may modulate the uncoupling of SAGA and TREX-2. We found that the total H2Bub1 level was unaltered in the rpt2-1 and SAC3-deleted strains (Supplementary Fig. S7), suggesting that the distribution of the functional Sgf73-DUBm may be essential for regulating $\mathrm{H} 2 \mathrm{Bub} 1$ levels near the ORF. $\mathrm{H} 2 \mathrm{~B}$ ubiquitylation is required for the efficient reassembly of nucleosomes during transcriptional elongation ${ }^{58,59}$, indicating that the Sgf73-DUBm may have an important role in $\mathrm{H} 2 \mathrm{Bub1-mediated} \mathrm{nucleosome}$ formation and stability. Thus, our findings should contribute to determining the specific links between the chromatin-modifying complex and the nuclear pore/nuclear transport machinery.

In addition to their involvement in the remodelling of the SAGA complex, the proteasomal ATPases were found to be critical for efficient mRNA export and the TREX-2-NPC association in vivo. We postulate that the 19S RP-mediated separation of the Sgf73DUBm is coupled with the mRNA export machinery during transcriptional elongation. The ubiquitylation/deubiquitylation of $\mathrm{H} 2 \mathrm{~B} / \mathrm{H} 2 \mathrm{Bub} 1$ in ORFs may be an important step in the dissociation of the TREX-2 complex from chromatin to the NPC. As Sgf73p recruits the TREX-2 complex to SAGA ${ }^{23}$, the Sgf73-DUBm may help to dissociate TREX-2, allowing it to interact with the NPC.

The targeting of SAGA to the promoter and the subsequent ATPase-mediated dissociation of the Sgf73-DUBm link the transcription of mRNA to its export from the nucleus to the cytoplasm (Fig. 7) ${ }^{60}$. Our findings raise the possibility that the

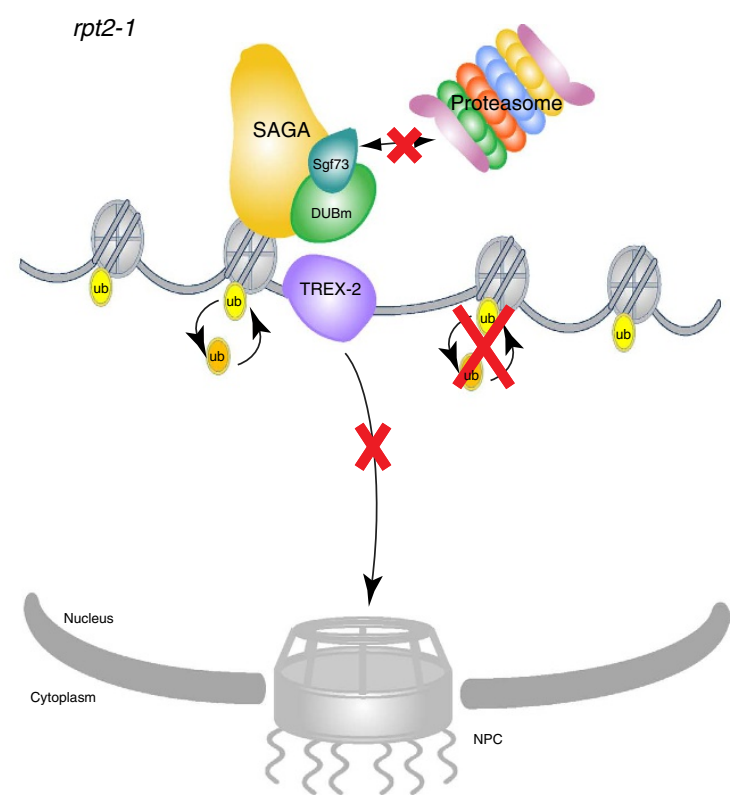

Figure 7 | Schematic representation of working model. The targeting of SAGA to the promoter and the subsequent dissociation of the Sgf73-DUBm by proteasomal ATPases links H2B ubiquitylation/deubiquitylation to mRNA export. The 19S RP remodels the SAGA complex using the proteasome's chaperone-like activity, resulting in the formation of a new functional subcomplex of SAGA, which we designated the 'Sgf73-DUBm'. Upon its dissociation, the Sgf73-DUBm may enter the ORF along with phosphorylated RNA Poll120,22. This is required for transcriptional elongation and is also important for the recycling of H2Bub1. As a result, the TREX-2 complex is dissociated from chromatin and targeted to the NPC by the Sgf73-DUBm. The chaperonelike activity of the proteasome is crucial for mRNA export, as the remodelling of the SAGA complex by the 19S RP is essential for the proper targeting of TREX-2 to the NPC. The rpt2-1 strain cannot establish specific binding between the proteasome and SAGA and is thus defective in mRNA export. 
proteasome-mediated separation of the chromatin-modifying complex into submodules may be a novel strategy for regulating the intricate patterns of eukaryotic gene expression.

\begin{abstract}
Methods
Yeast strains and protein purification. All of the strains used in this study are detailed in Supplementary Table S1. Genomic integration of the C-terminal (TAP, 13MYC, 3HA and GST) and the N-terminal (FLAG) tags and gene deletions were performed using the LiAc/SS-Carrier DNA/PEG method ${ }^{61}$ for yeast transformation via homologous recombination of PCR fragments. Yeast cells expressing TAPtagged protein were grown in Yeast Peptone Dextrose (YPD) at $30^{\circ} \mathrm{C}$ to an OD of about 1.0. The cell pellet was resuspended in Lysis buffer ( $40 \mathrm{mM}$ HEPES ( $\mathrm{pH} 7.5$ ), $350 \mathrm{mM} \mathrm{NaCl}, 10 \%$ Glycerol, $0.1 \%$ Tween-20, supplemented with a protease inhibitor) and disrupted using a bead beater (Biospec). Crude whole-cell extracts were subjected to ultracentrifugation, and the supernatant was directly applied to IgG-Sepharose (GE Healthcare) followed by overnight incubation at $4^{\circ} \mathrm{C}$ with $5 \mu \mathrm{g}$ of TEV. Three volume of calmodulin-binding buffer $(10 \mathrm{mM}$ Tris-Cl, $\mathrm{pH} 8.0,1 \mathrm{mM}$ Mg-Ac, $1 \mathrm{mM}$ imidazole, $2 \mathrm{mM} \mathrm{CaCl}, 0.1 \% \mathrm{NP}-40,10 \mathrm{mM} \beta$-mercaptoethanol, $300 \mathrm{mM} \mathrm{KCl}$ and $10 \%$ glycerol) was added to the TEV supernatant and incubated with Calmodulin Sepharose (Stratagene) for $3 \mathrm{~h}$. Purified complexes were resolved on $8 \%$ SDS-PAGE gels and visualized with silver staining.
\end{abstract}

GST pull-down analysis. GST-fusion proteins were bound to glutathione sepharose in PDB (150 mM NaCl, $50 \mathrm{mM}$ HEPES, pH 7.4, 0.1\% Tween-20, $10 \mathrm{mM}$ $\beta$-mercaptoethanol, $10 \%$ glycerol and $1 \mathrm{mM}$ PMSF). The binding reaction was conducted at $4^{\circ} \mathrm{C}$ in $0.5 \mathrm{ml}$ of PDB for $1 \mathrm{~h}$. The beads were washed five times in PDB at room temperature, boiled in Laemmli SDS sample buffer (4\% SDS, $20 \%$ glycerol, $10 \% \beta$-mercaptoethanol, $0.004 \%$ bromophenol blue and $0.125 \mathrm{M}$ Tris$\mathrm{HCl}$ ) and resolved using SDS-PAGE. The bound proteins were visualized using western blotting with antibodies against CBP and MYC.

In vitro analysis. For our in vitro SAGA remodelling assay, $25 \mu \mathrm{l}(\sim 250 \mathrm{ng})$ of the SAGA complexes from $w t$ and $r p t 2-1$ were incubated in 100- $\mu$ l reaction volumes containing $5 \mu \mathrm{l}(\sim 1 \mu \mathrm{g})$ of the $26 \mathrm{~S}$ proteasome (Fig. 1a) and the 19S RP from $w t$ and $r p t 2-1$ (Figs. 1b-c) or the lid and the base complexes from $w t$ (Fig. 1d) in reaction buffer (20 mM Tris-HCl, $\mathrm{pH} 8.0,25 \mathrm{mM} \mathrm{KCl}, 5 \mathrm{mM} \mathrm{MgCl}_{2}$ and $0.01 \%$ NP-40 with or without $2 \mathrm{mM} \mathrm{ATP}$ ) for $1 \mathrm{~h}$ at $30^{\circ} \mathrm{C}$ with constant mixing. After an additional incubation at $30^{\circ} \mathrm{C}$ for $10 \mathrm{~min}$ in $300 \mathrm{mM}+$ reaction buffer $(20 \mathrm{mM}$ Tris-HCl, $\mathrm{pH} 8.0,300 \mathrm{mM} \mathrm{KCl}, 5 \mathrm{mM} \mathrm{MgCl} 2$ and $0.01 \% \mathrm{NP}-40$ ), $40 \mu \mathrm{l}$ of calmodulin resin in calmodulin-binding buffer $(10 \mathrm{mM}$ Tris-Cl, $\mathrm{pH} 8.0,1 \mathrm{mM} \mathrm{Mg}-\mathrm{Ac}$, $1 \mathrm{mM}$ imidazole, $2 \mathrm{mM} \mathrm{CaCl}_{2}, 0.1 \% \mathrm{NP}-40,10 \mathrm{mM} \beta$-mercaptoethanol, $300 \mathrm{mM}$ $\mathrm{KCl}$ and $10 \%$ glycerol) was added to the reaction solution, and resin binding was performed at $4^{\circ} \mathrm{C}$ for $2 \mathrm{~h}$. Bead-bound and -unbound (supernatant) proteins were analysed with the help of western blotting on nitrocellulose membranes, using antibodies against CBP (Spt7p) (1:3,000), FLAG (Sgf73p) (1:2,000), MYC (Ubp8p) $(1: 1,000)$ and HA (Gen5p) $(1: 1,000)$. The antibodies against CBP (a polyclonal rabbit antibody), HA and MYC (monoclonal mouse antibodies) were produced inhouse. The monoclonal mouse anti-FLAG antibody was obtained from Sigma (F1804). Full scans of the western blots are shown in Supplementary Figs S8 and S9.

Deubiquitylation assay. The FLAG-tagged ubH2B substrate preparation was modified from a protocol described by Henry et al. ${ }^{20}$ Yeast cells expressing FLAGtagged $\mathrm{H} 2 \mathrm{~B}$ were grown in YPD at $30^{\circ} \mathrm{C}$ to an $\mathrm{OD}$ of about 1.0. Cells were collected and washed with $20 \%$ trichloroacetic acid (TCA). Cell pellet was resuspended in $0.5 \mathrm{ml}$ of $20 \%$ TCA and subjected to vortexing for $2 \mathrm{~min}$ with glass beads. The TCA pellet was resuspended in $0.2 \mathrm{ml}$ of $1 \mathrm{X}$ Laemmli sample buffer (2\% SDS, $60 \mathrm{mM}$ Tris-HCl, $\mathrm{pH} 6.8$ ) containing $\beta$-mercaptoethanol and unbuffered $2 \mathrm{M}$ Tris. The suspension was boiled for $3 \mathrm{~min}$ and centrifuged at 3,000 r.p.m. for $10 \mathrm{~min}$. The supernatant was added to IP buffer (50 mM Tris, pH 7.4, $150 \mathrm{mM}$ $\mathrm{NaCl}, 0.5 \% \mathrm{NP}-40$ and bovine serum albumin $\left.\left(0.5 \mathrm{mg} \mathrm{ml}^{-1}\right)\right)$ containing antiFLAG M2 affinity resin (Sigma). After mixing overnight at $4{ }^{\circ} \mathrm{C}$, the resin was washed with IP buffer. Substrates were eluted by incubation with $2 \mu$ of a $4 \mathrm{mg} \mathrm{ml}^{-1}$ solution of Flag peptide (Kodak) in IP buffer. The $500 \mathrm{ng}$ of substrate was incubated at $37^{\circ} \mathrm{C}$ for $5 \mathrm{~h}$ in DUB buffer $(100 \mathrm{mM}$ Tris- $\mathrm{HCl}, \mathrm{pH} 8.0,1 \mathrm{mM}$ EDTA, $1 \mathrm{mM}$ dithiothreitol, 5\% glycerol, $1 \mu \mathrm{M}$ PMSF and $1 \mu \mathrm{g} \mathrm{ml}^{-1}$ pepstatin A) containing $1 \%$ of the purified TAP-tagged proteins and various glycerol gradient fractions from $w t$ and $r p t 2-1$. The reaction was stopped on ice and run on a $16 \%$ SDS-PAGE gel. The resolved proteins were transferred to a nitrocellulose membrane, and western blot analysis was conducted using an antibody against HA.

LLVY assay. The purified proteasome samples were resolved by non-denaturing PAGE, as described in Glickman et al. ${ }^{62}$ Briefly, the samples were run on a $3.5 \%$ native polyacrylamide gel containing sample dye (xylene cyanol) in a cold room at $100 \mathrm{~V}$ for $3.5 \mathrm{~h}$. The gel was then incubated in resolving buffer $(90 \mathrm{mM}$ Tris base, $90 \mathrm{mM}$ boric acid, $5 \mathrm{mM} \mathrm{MgCl}, 0.5 \mathrm{mM}$ EDTA and $\left.1 \mathrm{mM} \mathrm{ATP}-\mathrm{MgCl}_{2}\right)$ containing fluorescent peptidase substrate (Suc-LLVY-AMC) with and without $0.02 \%$ SDS for $15 \mathrm{~min}$ at $30^{\circ} \mathrm{C}$. The proteasome bands were visualized under 380-nm UV light.
The liquid-based LLVY assay was performed according to the previously described method $^{42}$. The purified proteasome fractions were tested for peptidase activity with the fluorogenic substrate, Suc-LLVY-AMC, in assay buffer (50 mM Tris-HCl, $\mathrm{pH}$ 7.5, $40 \mathrm{mM} \mathrm{KCl}, 5 \mathrm{mM} \mathrm{MgCl}_{2}, 0.5 \mathrm{mM}$ ATP, $1 \mathrm{mM}$ dithiothreitol). Generation of free AMC was measured and quantified using a fluorescence spectrophotometer (Infinite 200; TECAN) and the Magellan 6 software (Magellan).

Assessing the accumulation of polyubiquitinated proteins. The wt and $r t p 2-1$ strains were grown in YPD medium at $30^{\circ} \mathrm{C}$ to an OD600 of $0.3-0.4$ and cultured in YPD at $37^{\circ} \mathrm{C}$. Cells were collected and lysed by the mild alkali method ${ }^{63}$. The extracts were subjected to SDS-PAGE, followed by western blotting with an antiFK2 antibody (04-263; Millipore) that specifically recognizes polyubiquitin chains ${ }^{64}$. Western blotting was also performed with an anti-H3 antibody (produced in-house) as a control.

Polyubiquitinated-Sic1 ${ }^{\text {PY }}$ degradation assay. The PY motif was introduced into the N-terminal region of Sicl to generate Sicl ${ }^{\mathrm{PY}}$, which allows Rsp 5 to bind to Sic1. To prepare polyubiquitinated Sic1 ${ }^{\text {PY } 65}$, mix 1 , which is composed of $4.5 \mu \mathrm{l}$ of buffer A (50 mM Tris-HCl, pH 7.5, $100 \mathrm{mM} \mathrm{NaCl}$, and $10 \%$ glycerol), $2 \mu \mathrm{l}$ of $5 \times \mathrm{ATP}$ solution, $0.5 \mu \mathrm{l}$ of Uba1 (E1), $1 \mu \mathrm{l}$ of Ubc4 (E4), $2 \mu \mathrm{l}$ of ubiquitin, was preincubated for $10 \mathrm{~min}$ at $25^{\circ} \mathrm{C}$. After preincubation of mix 1, mix 2, which is composed of $6 \mu \mathrm{l}$ of buffer A, $2 \mu$ l of $5 \times$ ATP solution of Rsp5 (E3) and $1 \mu$ l of Sicl ${ }^{\text {PY }}$, was mixed with mixl and incubated for $3 \mathrm{~h}$ at $25^{\circ} \mathrm{C}$. The polyubiquitinated Sicl ${ }^{\mathrm{PY}}$ was incubated with affinity-purified $26 \mathrm{~S}$ proteasomes from $w t$ and $r p t 2-1$ at $25^{\circ} \mathrm{C}$. The reaction was stopped by the addition of SDS-loading buffer, and the samples were resolved using SDS-PAGE and subjected to western blotting with an anti-T7 antibody (69048-3; Novagen) to assess degradation.

Chromatin immunoprecipitation. ChIP was performed as previously reported ${ }^{66}$. Yeast cells were grown in YPD at $30^{\circ} \mathrm{C}$ to an OD600 of 0.8 , followed by cross-linking and sonication. The resulting extracts were immunoprecipitated with antibodies against MYC (a polyclonal rabbit antibody), 8WG16 (ab817; Abcam) and the other indicated proteins (polyclonal rabbit antibodies). Immunoprecipitation was repeated with at least three different extracts per condition. The immunoprecipitated DNA was analysed using quantitative real-time PCR using the CFX96 Real-Time System (Bio-Rad). The cycle thresholds for the target genes were calculated relative to those of the intergenic region on chromosome $\mathrm{V}$. The primers used for PCR are listed in Supplementary Table S2.

Fluorescence imaging and FISH analysis. Prior to live-cell imaging, cells were grown to the mid-log phase in YPD (Figs 5b,c, 6a). Fluorescence was assessed using an Image M1 fluorescence microscope equipped with a $\times 100 \mathrm{NA} 1.3$ oil immersion lens and GFP, $4^{\prime}, 6$-diamidino-2-phenylindole dihydrochloride and $\mathrm{Cy} 3$ filters (all from Carl Zeiss, Inc.). Images were acquired using an Axio Cam HR camera and the Axio Vision 4.3 software (Carl Zeiss, Inc.) at a resolution of $1,388 \times 1,040$ (standard mono) at $25^{\circ} \mathrm{C}$. To perform in situ hybridization of poly (A) + RNA $^{23}$, cells were grown to an OD600 of $0.3-0.4$ at $30^{\circ} \mathrm{C}$ (Fig. $5 \mathrm{c}$ ) and fixed using $4 \%$ paraformaldehyde in $100 \mathrm{mM}$ phosphate buffer $(\mathrm{pH} 6.5)$ for $3 \mathrm{~h}$ at $25^{\circ} \mathrm{C}$. Cells were added to $0.5 \mathrm{ml}$ of $1.2 \mathrm{M}$ sorbitol in $0.1 \mathrm{M}$ phosphate buffer containing $\beta$-mercaptoethanol to form spheroplasts and were incubated for $20 \mathrm{~min}$. Finally, nuclei were counterstained with $0.1 \mu \mathrm{g} \mathrm{ml}^{-1} 4^{\prime}, 6$-diamidino-2-phenylindole dihydrochloride.

\section{References}

1. Coux, O., Tanaka, K. \& Goldberg, A. L. Structure and functions of the $20 \mathrm{~S}$ and 26S proteasomes. Annu. Rev. Biochem. 65, 801-847 (1996).

2. Ferrell, K., Wilkinson, C. R., Dubiel, W. \& Gordon, C. Regulatory subunit interactions of the $26 \mathrm{~S}$ proteasome, a complex problem. Trends Biochem. Sci. 25, 83-88 (2000).

3. Braun, B. C. et al. The base of the proteasome regulatory particle exhibits chaperone-like activity. Nat. Cell Biol. 1, 221-226 (1999).

4. Liu, C.-W. et al. Conformational remodeling of proteasomal substrates by PA700, the $19 \mathrm{~S}$ regulatory complex of the $26 \mathrm{~S}$ proteasome. J. Biol. Chem. 277, 26815-26820 (2002)

5. Strickland, E., Hakala, K., Thomas, P. J. \& DeMartino, G. N. Recognition of misfolding proteins by PA700, the regulatory subcomplex of the $26 \mathrm{~S}$ proteasome. J. Biol. Chem. 275, 5565-5572 (2000).

6. Demartino, G. N. \& Gillette, T. G. Proteasomes: machines for all reasons. Cell 129, 659-662 (2007)

7. Nishiyama, A. A nonproteolytic function of the proteasome is required for the dissociation of $\mathrm{Cdc} 2$ and cyclin B at the end of M phase. Genes Dev. 14, 2344-2357 (2000).

8. Keppler, B. R., Archer, T. K. \& Kinyamu, H. K. Emerging roles of the $26 \mathrm{~S}$ proteasome in nuclear hormone receptor-regulated transcription. Biochim. Biophys. Acta. 1809, 109-118 (2011).

9. Kwak, J., Workman, J. L. \& Lee, D. The proteasome and its regulatory roles in gene expression. Biochim. Biophys. Acta. 1809, 88-96 (2010). 
10. Ezhkova, E. \& Tansey, W. P. Proteasomal ATPases link ubiquitylation of histone H2B to methylation of histone H3. Mol. Cell 13, 435-442 (2004).

11. Ferdous, A., Gonzalez, F., Sun, L., Kodadek, T. \& Johnston, S. A. The 19 S regulatory particle of the proteasome is required for efficient transcription elongation by RNA polymerase II. Mol. Cell 7, 981-991 (2001).

12. Xu, Q., Singer, R. \& Johnston, G. C. Sug1 modulates yeast transcription activation by Cdc68. Mol. Cell. Biol. 15, 6025-6035 (1995).

13. Malik, S., Shukla, A., Sen, P. \& Bhaumik, S. R. The 19 S proteasome subcomplex establishes a specific protein interaction network at the promoter for stimulated transcriptional initiation in vivo. J. Biol. Chem. 284, 35714-35724 (2009).

14. Lee, D. et al. The proteasome regulatory particle alters the SAGA coactivator to enhance its interactions with transcriptional activators. Cell 123, 423-436 (2005).

15. Daniel, J. A. \& Grant, P. A. Multi-tasking on chromatin with the SAGA coactivator complexes. Mutat. Res. 618, 135-148 (2007).

16. Grant, P. A. et al. Yeast Gen5 functions in two multisubunit complexes to acetylate nucleosomal histones: characterization of an Ada complex and the SAGA (Spt/Ada) complex. Genes Dev. 11, 1640-1650 (1997).

17. Baker, S. P. \& Grant, P. A. The SAGA continues: expanding the cellular role of a transcriptional co-activator complex. Oncogene 26, 5329-5340 (2007).

18. Rodríguez-Navarro, S. Insights into SAGA function during gene expression. EMBO. Rep. 10, 843-850 (2009).

19. Koutelou, E., Hirsch, C. L. \& Dent, S. Y. R. Multiple faces of the SAGA complex. Curr. Opin. Cell Biol. 22, 374-382 (2010).

20. Henry, K. W. et al. Transcriptional activation via sequential histone $\mathrm{H} 2 \mathrm{~B}$ ubiquitylation and deubiquitylation, mediated by SAGA-associated Ubp8. Genes Dev. 17, 2648-2663 (2003).

21. Daniel, J. A. et al. Deubiquitination of histone H2B by a yeast acetyltransferase complex regulates transcription. J. Biol. Chem. 279, 1867-1871 (2004).

22. Wyce, A. et al. H2B ubiquitylation acts as a barrier to Ctk1 nucleosomal recruitment prior to removal by Ubp8 within a SAGA-related complex. Mol. Cell 27, 275-288 (2007).

23. Köhler, A., Schneider, M., Cabal, G. G., Nehrbass, U. \& Hurt, E. Yeast Ataxin-7 links histone deubiquitination with gene gating and mRNA export. Nat. Cell Biol. 10, 707-715 (2008).

24. Mannen, T., Andoh, T. \& Tani, T. Dss1 associating with the proteasome functions in selective nuclear mRNA export in yeast. Biochem. Biophys. Res. Commun. 365, 664-671 (2008).

25. Ellisdon, A. M., Dimitrova, L., Hurt, E. \& Stewart, M. Structural basis for the assembly and nucleic acid binding of the TREX-2 transcription-export complex. Nat. Struct. Mol. Biol. 19, 328-336 (2012).

26. Faza, M. B. et al. Seml is a functional component of the nuclear pore complexassociated messenger RNA export machinery. J. Cell Biol. 184, 833-846 (2009).

27. Wilmes, G. M. et al. A genetic interaction map of RNA-processing factors reveals links between Sem1/Dss1-containing complexes and mRNA export and splicing. Mol. Cell 32, 735-746 (2008).

28. Swaminathan, S. A. SAGA of proteasomal ATPases. Nat. Cell Biol. 8, 13 (2006).

29. Matilla, A. et al. Association of ataxin-7 with the proteasome subunit S4 of the 19S regulatory complex. Hum. Mol. Genet. 10, 2821-2831 (2001).

30. Groll, M. Structure of 20 S proteasome from yeast at 2.4. Nature 386, 463-471 (1997).

31. Glickman, M. H., Rubin, D. M. \& Fried, V. A. The regulatory particle of the Saccharomyces cerevisiae proteasome. Mol. Cell Biol. 18, 3149-3162 (1998).

32. Glickman, M. H. et al. A subcomplex of the proteasome regulatory particle required for ubiquitin-conjugate degradation and related to the COP9signalosome and eIF3. Cell 94, 615-623 (1998).

33. Grant, P. A. et al. A subset of TAF(II)s are integral components of the SAGA complex required for nucleosome acetylation and transcriptional stimulation. Cell 94, 45-53 (1998).

34. Mcmahon, S. J., Pray-grant, M. G., Schieltz, D., Iii, J. R. Y. \& Grant, P. A Polyglutamine-expanded spinocerebellar ataxia-7 protein disrupts normal SAGA and SLIK histone acetyltransferase activity. Proc. Natl Acad. Sci. USA 102, 8478-8482 (2005).

35. Lee, K. K., Swanson, S. K., Florens, L., Washburn, M. P. \& Workman, J. L. Yeast Sgf73/Ataxin-7 serves to anchor the deubiquitination module into both SAGA and Slik(SALSA) HAT complexes. Epigenet. Chromatin 2, 2 (2009).

36. Samara, N. L. et al. Structural insights into the assembly and function of the SAGA deubiquitinating module. Science 328, 1025-1029 (2010).

37. Köhler, A., Zimmerman, E., Schneider, M., Hurt, E. \& Zheng, N. Structural basis for assembly and activation of the heterotetrameric SAGA histone $\mathrm{H} 2 \mathrm{~B}$ deubiquitinase module. Cell 141, 606-617 (2010).

38. Eberharter, A., John, S., Grant, P. A., Utley, R. T. \& Workman, J. L. Identification and analysis of yeast nucleosomal histone acetyltransferase complexes. Methods 15, 315-321 (1998).

39. Heinemeyer, W., Gruhler, A., Möhrle, V., Mahé, Y. \& Wolf, D. H. PRE2, highly homologous to the human major histocompatibility complex-linked RING10 gene, codes for a yeast proteasome subunit necessary for chrymotryptic activity and degradation of ubiquitinated proteins. J. Biol. Chem. 268, 5115-5120 (1993).
40. Chen, P. \& Hochstrasser, M. Active site formation in the 20 S proteasome to completion of assembly. Cell 86, 961-972 (1996).

41. Elsasser, S., Schmidt, M. \& Finley, D. Characterization of the proteasome using native gel electrophoresis. Methods Enzymol. 398, 353-363 (2005).

42. Kisselev, A. F. \& Goldberg, A. L. Monitoring activity and inhibition of $26 \mathrm{~S}$ proteasomes with fluorogenic peptide substrates. Methods Enzymol. 398, 364-378 (2005).

43. Ghislain, M., Udvardy, A. \& Mann, C. S. cerevisiae 26S protease mutants arrest cell division in G2/metaphase. Nature 366, 358-362 (1993).

44. Verma, R. et al. Proteasomal proteomics: identification of nucleotide-sensitive proteasome-interacting proteins by mass spectrometric analysis of affinitypurified proteasomes. Mol. Biol. Cell 11, 3425-3439 (2000).

45. Verma, R. et al. Role of Rpn11 metalloprotease in deubiquitination and degradation by the 26S proteasome. Science 298, 611-615 (2002).

46. Thompson, N. E., Steinberg, T. H., Aronson, D. B. \& Burgess, R. R. Inhibition of in vivo and in vitro transcription by monoclonal antibodies prepared against wheat germ RNA polymerase II that react with the heptapeptide repeat of eukaryotic RNA polymerase II. J. Biol. Chem. 264, 11511-11520 (1989).

47. Köhler, A. \& Hurt, E. Exporting RNA from the nucleus to the cytoplasm. Nat. Rev. Mol. Cell. Biol. 8, 761-773 (2007).

48. Fischer, T. The mRNA export machinery requires the novel Sac3p-Thplp complex to dock at the nucleoplasmic entrance of the nuclear pores. EMBO J. 21, 5843-5852 (2002).

49. Huertas, P. \& Luna, R. The THP1-SAC3-SUS1-CDC31 complex works in transcription elongation-mRNA export preventing RNA-mediated genome instability. Mol. Biol. Cell 19, 4310-4318 (2008).

50. Rodríguez-Navarro, S. et al. Sus1, a functional component of the SAGA histone acetylase complex and the nuclear pore-associated mRNA export machinery. Cell 116, 75-86 (2004).

51. Lei, E. P. et al. Sac3 Is an mRNa export factor that localizes to cytoplasmic fibrils of nuclear pore complex. Mol. Biol. Cell 14, 836-847 (2003).

52. Cabal, G. G. et al. SAGA interacting factors confine sub-diffusion of transcribed genes to the nuclear envelope. Nature 441, 770-773 (2006).

53. Kirchenbauer, M. \& Liakopoulos, D. An auxiliary, membrane-based mechanism for nuclear migration in budding yeast. Mol. Biol. Cell 24, 1434-1443 (2013).

54. Lei, E. P. \& Silver, P. A. Protein and RNA export from the nucleus. Dev. Cell 2, 261-272 (2002).

55. Strässer, K. \& Hurt, E. Yralp, a conserved nuclear RNA-binding protein, interacts directly with Mex67p and is required for mRNA export. EMBO J. 19, 410-420 (2000).

56. Segref, A. et al. Mex67p, a novel factor for nuclear mRNA export, binds to both poly(A) + RNA and nuclear pores. EMBO J. 16, 3256-3271 (1997).

57. Santos-Rosa, H. et al. Nuclear mRNA export requires complex formation between Mex67p and Mtr2p at the nuclear pores. Mol. Cell Biol. 18, 6826-6838 (1998).

58. Batta, K., Zhang, Z., Yen, K., Goffman, D. B. \& Pugh, B. F. Genome-wide function of H2B ubiquitylation in promoter and genic regions. Genes Dev. 25, 2254-2265 (2011).

59. Fleming, A. B., Kao, C.-F., Hillyer, C., Pikaart, M. \& Osley, M. A. H2B ubiquitylation plays a role in nucleosome dynamics during transcription elongation. Mol. Cell 31, 57-66 (2008).

60. Vitaliano-Prunier, A. et al. H2B ubiquitylation controls the formation of export-competent mRNP. Mol. Cell 45, 132-139 (2012).

61. Gietz, R. D. \& Woods, R. a Transformation of yeast by lithium acetate/singlestranded carrier DNA/polyethylene glycol method. Methods Enzymol. 350, 87-96 (2002).

62. Glickman, M. H., Rubin, D. M., Fried, V. A. \& Finley, D. The regulatory particle of the Saccharomyces cerevisiae proteasome. Mol. Cell. Biol. 18, 3149-3162 (1998).

63. Kushnirov, V. V. Rapid and reliable protein extraction from yeast. Yeast 16, 857-860 (2000).

64. Fujimuro, M., Sawada, H. \& Yokosawa, H. Production and characterization of monoclonal antibodies specific to multi-ubiquitin chains of polyubiquitinated proteins. FEBS Lett. 349, 173-180 (1994).

65. Saeki, Y., Isono, E. \& Toh-E, A. Preparation of ubiquitinated substrates by the PY motif-insertion method for monitoring 26S proteasome activity. Methods Enzymol. 399, 215-227 (2005).

66. Gonzalez, F., Delahodde, A., Kodadek, T. \& Johnston, S. A. Recruitment of a 19S proteasome subcomplex to an activated promoter. Science 296, 548-550 (2002).

67. Rigaut, G. et al. A generic protein purification method for protein complex characterization and proteome exploration. Nat. Biotechnol. 17, 1030-1032 (1999).

\section{Acknowledgements}

We thank Dr W.K. Huh (Seoul National University) for providing the Sac3-GFPexpressing strain, Dr Ed Hurt (Biochemie-Zentrum der Universität Heidelberg) for providing the DNA constructs encoding MEX67, MTR2, mex67-5 and mtr2-9, and Dr Y. Saeki (Tokyo Metropolitan Institute of Medical Science) for providing the DNA 
constructs encoding Sic1 ${ }^{\mathrm{PY}}$, E2 and E3. We also thank Drs. Jerry L. Workman (Stowers Institute for Medical Research) and William P. Tansey (Vanderbilt University School of Medicine) for their critical reading and suggestions on our initial manuscript. This work was supported by the Stem Cell Research Program (2012M 3A9B 4027953) and by a grant from the KAIST Future Systems Healthcare Project, Ministry of Science, ICT and Future Planning, South Korea.

\section{Author contributions}

S.L., J.K. and M.K. designed and performed the experiments, with suggestions from D.L.; S.L. and M.K. imaged and analysed the data from all experiments; and S.L., M.K. and D.L. wrote the manuscript.

\section{Additional information}

Supplementary Information accompanies this paper at http://www.nature.com/ naturecommunications

Competing financial interests: The authors declare no competing financial interests.

Reprints and permission information is available online at http://npg.nature.com/ reprintsandpermissions/

How to cite this article: Lim, S. et al. Separation of a functional deubiquitylating module from the SAGA complex by the proteasome regulatory particle. Nat. Commun. 4:2641 doi: 10.1038/ncomms3641 (2013). 